ESAIM: M2AN 52 (2018) 2003-2035

https://doi.org/10.1051/m2an/2018011
ESAIM: Mathematical Modelling and Numerical Analysis

www.esaim-m2an.org

\title{
NUMERICAL ANALYSIS OF THE MIXED FINITE ELEMENT METHOD FOR THE NEUTRON DIFFUSION EIGENPROBLEM WITH HETEROGENEOUS COEFFICIENTS
}

\author{
P. Ciarlet Jr. ${ }^{1}$, L. Giret ${ }^{1,2}$, E. Jamelot ${ }^{3, *}$ and F.D. Kpadonou ${ }^{1,4}$
}

\begin{abstract}
We study first the convergence of the finite element approximation of the mixed diffusion equations with a source term, in the case where the solution is of low regularity. Such a situation commonly arises in the presence of three or more intersecting material components with different characteristics. Then we focus on the approximation of the associated eigenvalue problem. We prove spectral correctness for this problem in the mixed setting. These studies are carried out without, and then with a domain decomposition method. The domain decomposition method can be non-matching in the sense that the traces of the finite element spaces may not fit at the interface between subdomains. Finally, numerical experiments illustrate the accuracy of the method.
\end{abstract}

Mathematics Subject Classification. 65N25, 65N30, 82D75

Received July 7, 2017. Accepted January 25, 2018.

\section{INTRODUCTION}

The multigroup neutron diffusion equation, which is an approximation of the multigroup neutron transport equation, is important in nuclear industry since it allows to model many nuclear reactor cores [16]. In the steady state case, it corresponds to a generalized eigenvalue problem. We propose here the numerical analysis of this problem in the case of a discretization with mixed finite elements, possibly with a domain decomposition method. We focus on the one group of energy case which is the base block of the multigroup case. This paper is thus the extension of [13], where the authors proposed the numerical analysis of the one-group neutron diffusion equation with a source term, discretized with mixed finite elements, with matching and non-matching domain decomposition methods.

Nuclear reactor cores often have a Cartesian geometry. In Figure 1a, we draw a top view of a PWR-like core model. Each square, which represents a part of the reflector or an assembly, is made itself of cells, which are rectangular cuboids of $\mathbb{R}^{3}$. In Figure 1 b, we make a zoom on a patch of six $(3 \times 2)$ assemblies: each

Keywords and phrases: Diffusion equation, low-regularity solution, mixed formulation, eigenproblem, domain decomposition methods.

1 POEMS, ENSTA ParisTech, CNRS, INRIA, 828 Bd des Maréchaux, 91762 Palaiseau Cedex, France.

2 DEN-Service d'Etudes des Réacteurs et de Mathématiques Appliquées-SERMA, LLPR, CEA, Université Paris-Saclay, 91191 Gif-sur-Yvette Cedex, France.

${ }^{3}$ DEN-Service de Thermo-hydraulique et de Mécanique des Fluides-STMF, LMSF, CEA, Université Paris-Saclay, 91191 Gif-sur-Yvette Cedex, France.

${ }^{4}$ Laboratoire de Mathématiques de Versailles, UVSQ, 45 Av des Etats-Unis, 78035 Versailles Cedex, France.

* Corresponding author: erell.jamelot@cea.fr 


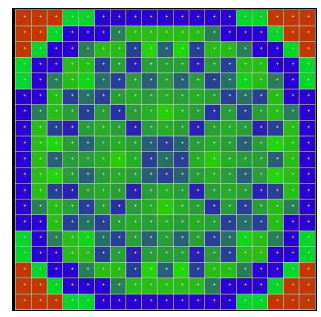

(a) Reactor core model

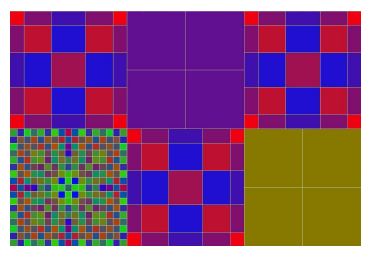

(b) Patch of six assemblies

Figure 1. 2D depiction of a PWR core and a zoom on six assemblies.

colored square represents a cell containing fuel, absorbing or reflector material. In our model, the coefficients are polynomial (possibly constant) in each cell [16, 23, 24]. The global domain of the reactor core (see again Fig. 1a) is represented by a rectangular cuboid of $\mathbb{R}^{3}$. In practice the coefficients characterizing the materials may differ from one cell to another by a factor of order 10 or more.

The outline is as follows. In Section 2, we introduce the notations, and recall basic mathematical definitions. In the next section, we provide the main abstract tool that enables us to characterize the so-called low-regularity solutions, that is piecewise $H^{1+r}$ solutions with an exponent $r>0$ that can be (arbitrarily) small. Then in Section 4, we solve the diffusion equation written in mixed form, with either a source term, or as an eigenproblem. We recall that the approximation of eigenvalue problems has been studied among others by Osborn et al. in [1, 27], and in particular by Boffi et al. [4, 5, 6] when the eigenproblem is in a mixed form. In our case however, their theory does not ensure the spectral correctness of the approximation so we design a new proof to obtain this result. On the other hand, we can adapt the work of Boffi et al. [8] to exhibit a convergence rate for the eigenvalues. For the discretization, we choose the well-known Raviart-Thomas-Nédélec finite element. Then in Sections 5 and 6, we consider the same problems, solved now with the help of a Domain Decomposition method: the $\mathrm{DD}+L^{2}$-jumps method. Finally, we analyze the numerical capabilities of the $\mathrm{DD}+L^{2}$-jumps method, before giving some concluding remarks.

\section{Geometry, Hilbert spaces and notations}

Throughout the paper, $C$ is used to denote a generic positive constant which is independent of the meshsize, the triangulation and the quantities/fields of interest. We also use the shorthand notation $A \lesssim B$ for the inequality $A \leq C B$, where $A$ and $B$ are two scalar quantities, and $C$ is a generic constant. Respectively, $A \approx B$ for the inequalities $A \lesssim B$ and $B \lesssim A$.

Vector-valued (resp. tensor-valued) function spaces are written in boldface character (resp. blackboard characters); for the latter, the index sym indicates symmetric fields. Given an open set $O \in \mathbb{R}^{d}, d=1,2,3$, we use the notation $(\cdot \mid \cdot)_{0, O}$ (respectively $\left.\|\cdot\|_{0, O}\right)$ for the $L^{2}(O)$ and $\mathbf{L}^{2}(O):=\left(L^{2}(O)\right)^{d}$ scalar products (resp. norms). More generally, $(\cdot \mid \cdot)_{s, O}$ and $\|\cdot\|_{s, O}$ (respectively $|\cdot|_{s, O}$ ) denote the scalar product and norm (resp. semi-norm) of the Sobolev spaces $H^{s}(O)$ and $\mathbf{H}^{s}(O):=\left(H^{s}(O)\right)^{d}$ for $s \in \mathbb{R}$ (resp. for $s>0$ ).

If moreover the boundary $\partial O$ is Lipschitz, $\mathbf{n}$ denotes the unit outward normal vector field to $\partial O$. Finally, it is assumed that the reader is familiar with vector-valued function spaces related to the diffusion equation, such as $\mathbf{H}(\operatorname{div} ; O), \mathbf{H}_{0}(\operatorname{div} ; O)$, etc.

We let $\mathcal{R}$ be a bounded, connected and open subset of $\mathbb{R}^{d}$, having a Lipschitz boundary which is piecewise smooth. We split $\mathcal{R}$ into $N$ open disjoint parts $\left\{\mathcal{R}_{i}\right\}_{1 \leq i \leq N}$ with Lipschitz, piecewise smooth boundaries: $\overline{\mathcal{R}}=$ $\cup_{1 \leq i \leq N} \overline{\mathcal{R}_{i}}$ and the set $\left\{\mathcal{R}_{i}\right\}_{1 \leq i \leq N}$ is called a partition of $\mathcal{R}$. For a field $v$ defined over $\mathcal{R}$, we shall use the notations $v_{i}=v_{\mid \mathcal{R}_{i}}$, for $1 \leq i \leq N$. 
Given a partition $\left\{\mathcal{R}_{i}\right\}_{1 \leq i \leq N}$ of $\mathcal{R}$, we introduce function spaces with piecewise regular elements:

$$
\begin{aligned}
\mathcal{P} H^{s}(\mathcal{R}) & =\left\{\psi \in L^{2}(\mathcal{R}) \mid \psi_{i} \in H^{s}\left(\mathcal{R}_{i}\right), 1 \leq i \leq N\right\}, s>0 ; \\
\mathcal{P} W^{1, \infty}(\mathcal{R}) & =\left\{D \in L^{\infty}(\mathcal{R}) \mid D_{i} \in W^{1, \infty}\left(\mathcal{R}_{i}\right), 1 \leq i \leq N\right\} .
\end{aligned}
$$

We recall that for a piecewise smooth $\psi \in \mathcal{P} H^{s}(\mathcal{R}),\|\psi\|_{\mathcal{P} H^{s}(\mathcal{R})}^{2}=\sum_{i=1}^{N}\|\psi\|_{s, \mathcal{R}_{i}}^{2}$. Similarly for elements of $\mathcal{P} W^{1, \infty}(\mathcal{R})$.

\section{Setting OF THE MODEL}

Given a source term $S_{f} \in L^{2}(\mathcal{R})$, we consider the following neutron diffusion equation, with vanishing Dirichlet boundary condition. In its primal form, it is written:

Find $\phi \in H_{0}^{1}(\mathcal{R})$ such that:

$$
-\operatorname{div} D \operatorname{grad} \phi+\Sigma_{a} \phi=S_{f} \text { in } \mathcal{R}
$$

where $\phi, D$, and $\Sigma_{a}$ denote respectively the neutron flux, the diffusion coefficient and the macroscopic absorption cross section. Finally, $S_{f}$ denotes the fission source. When $S_{f}$ depends on $\phi$, the steady state neutron diffusion equation is a generalized eigenvalue problem. It reads (one group of energy):

Find $\phi \in H_{0}^{1}(\mathcal{R}) \backslash\{0\}, \lambda \in \mathbb{R}$ such that:

$$
-\operatorname{div} D \operatorname{grad} \phi+\Sigma_{a} \phi=\lambda \underline{\nu \Sigma_{f}} \phi \text { in } \mathcal{R}
$$

where $\underline{\nu \Sigma_{f}}$ is the fission yield times the macroscopic fission cross section. Under the assumption that the coefficients $D, \Sigma_{a}$ and $\underline{\nu \Sigma_{f}}$ are positive, the physical solution corresponds to the smallest $\lambda \geq 0[12,16]$. When this problem is solved using the inverse power iteration, the source problem (3.1) corresponds to one iteration step, which further justifies its study.

When solving the neutron diffusion equation, $D$ is scalar-valued. From now on and unless otherwise specified, we adopt the more general setting of a (symmetric) tensor-valued coefficient $D$. The coefficients defining problems (3.1) and (3.2) satisfy the assumptions:

$$
\left\{\begin{array}{l}
\left(D, \Sigma_{a}, \underline{\nu \Sigma_{f}}\right) \in \mathbb{L}_{\text {sym }}^{\infty}(\mathcal{R}) \times L^{\infty}(\mathcal{R}) \times L^{\infty}(\mathcal{R}), \\
\exists D_{*}, D^{*}>0, \forall \boldsymbol{z} \in \mathbb{R}^{d}, D_{*}\|\boldsymbol{z}\|^{2} \leq(D \boldsymbol{z}, \boldsymbol{z}) \leq D^{*}\|\boldsymbol{z}\|^{2} \text { a.e. in } \mathcal{R}, \\
\exists\left(\Sigma_{a}\right)_{*},\left(\Sigma_{a}\right)^{*}>0,0<\left(\Sigma_{a}\right)_{*} \leq \Sigma_{a} \leq\left(\Sigma_{a}\right)^{*} \text { a.e. in } \mathcal{R}, \\
0 \leq{\underline{\nu \Sigma_{f}}}_{\text {a.e. in } \mathcal{R}, \underline{\nu \Sigma_{f}} \neq 0 .}
\end{array}\right.
$$

In particular, it can happen that $\underline{\nu \Sigma_{f}}$ vanishes on some regions. Also, it is well known that problem (3.1) is equivalent to the following variational formulation:

Find $\phi \in H_{0}^{1}(\mathcal{R})$ such that $\forall \psi \in H_{0}^{1}(\mathcal{R})$ :

$$
\int_{\mathcal{R}} D \operatorname{grad} \phi \cdot \operatorname{grad} \psi+\int_{\mathcal{R}} \Sigma_{a} \phi \psi=\int_{\mathcal{R}} S_{f} \psi .
$$

Under the assumptions (3.3) on the coefficients, the primal problem (3.1) is well-posed, in the sense that for all $S_{f} \in L^{2}(\mathcal{R})$, there exists one and only one $\phi \in H_{0}^{1}(\mathcal{R})$ that solves (3.1), and in addition there holds $\|\phi\|_{1, \mathcal{R}} \lesssim\left\|S_{f}\right\|_{0, \mathcal{R}}$. We recall that under additional mild assumptions on the coefficients, the solution $\phi$ has some extra regularity (see [9, 14] and [13], Prop. 1). 
Proposition 3.1. Let $D \in \mathcal{P} \mathbb{W}_{\text {sym }}^{1, \infty}(\mathcal{R})$ and $\Sigma_{a} \in \mathcal{P} W^{1, \infty}(\mathcal{R})$ satisfy $(3.3)$. There exists $\left.\left.r_{\max } \in\right] 0,1\right]$, called the regularity exponent, such that for all source terms $S_{f} \in L^{2}(\mathcal{R})$, the solution $\phi \in H^{1}(\mathcal{R})$ to problem (3.1) belongs to $\bigcap_{0<r<r_{\max }} \mathcal{P} H^{1+r}(\mathcal{R})\left(r_{\max }<1\right)$ or $\mathcal{P} H^{2}(\mathcal{R})\left(r_{\max }=1\right)$ with continuous dependence:

$\forall r \in\left[0, r_{\max }\left[,\|\phi\|_{\mathcal{P} H^{1+r}(\mathcal{R})} \lesssim\left\|S_{f}\right\|_{0, \mathcal{R}}\left(r_{\max }<1\right)\right.\right.$ or $\|\phi\|_{\mathcal{P} H^{2}(\mathcal{R})} \lesssim\left\|S_{f}\right\|_{0, \mathcal{R}}\left(r_{\max }=1\right)$.

In the following, we study the two different problems, the source problem (3.1) and the eigenvalue problem (3.2). Unless otherwise specified, we keep the assumptions of Proposition 3.1 throughout the paper. Since crosspoints are allowed in our model, $c f$. Figure 1a, and in accordance with [9], the low-regularity case corresponds precisely to

$$
r_{\max }<1 / 2
$$

For the eigenvalue problem, the analysis is carried out for eigenfunctions which can be either low-regularity functions or "smooth" functions.

Remark 3.2. Instead of imposing a vanishing Dirichlet boundary condition in the model, one can consider a vanishing Neumann boundary condition $D \operatorname{grad} \phi \cdot \mathbf{n}=0$ on $\partial \mathcal{R}$. Under some slight restrictions on the geometry, one can also consider a vanishing Fourier boundary condition $\mu_{F} \phi+D \operatorname{grad} \phi \cdot \mathbf{n}=0$ on $\partial \mathcal{R}$, with $\mu_{F}>0$. In the latter case, the restriction is that the coefficient $D$ is smooth in a neighborhood of the boundary. The theory and numerical analysis written hereafter still apply.

\section{THE PLAIN CASE}

We start our study with the neutron diffusion problem without domain decomposition method: we call it the plain case. In this section, we use the function space:

$$
\mathbf{X}=\left\{\xi:=(\mathbf{q}, \psi) \in \mathbf{H}(\operatorname{div}, \mathcal{R}) \times L^{2}(\mathcal{R})\right\}, \quad\|\xi\|_{\mathbf{X}}:=\left(\|\mathbf{q}\|_{\mathbf{H}(\operatorname{div}, \mathcal{R})}^{2}+\|\psi\|_{0, \mathcal{R}}^{2}\right)^{1 / 2}
$$

From now on, we use the notations: $\zeta=(\mathbf{p}, \phi)$ and $\xi=(\mathbf{q}, \psi)$.

\subsection{Setting of the mixed variational formulation}

Starting from the solution $\phi$ to (3.1), if one lets $\mathbf{p}:=-D \operatorname{grad} \phi \in \mathbf{L}^{2}(\mathcal{R})$, known as the neutron current, one may write the neutron diffusion problem as:

Find $(\mathbf{p}, \phi) \in \mathbf{H}(\operatorname{div}, \mathcal{R}) \times H_{0}^{1}(\mathcal{R})$ such that:

$$
\begin{cases}-D^{-1} \mathbf{p}-\operatorname{grad} \phi=0 & \text { in } \mathcal{R} \\ \operatorname{div} \mathbf{p}+\Sigma_{a} \phi=S_{f} & \text { in } \mathcal{R} .\end{cases}
$$

Solving the mixed problem (4.1) is actually equivalent to solving (3.1), as the result below recalls.

Theorem 4.1. Let $D$ satisfy (3.3). The solution $(\mathbf{p}, \phi) \in \mathbf{H}(\operatorname{div}, \mathcal{R}) \times H_{0}^{1}(\mathcal{R})$ to (4.1) is such that $\phi$ is a solution to (3.1) with the same data. Conversely, the solution $\phi \in H_{0}^{1}(\mathcal{R})$ to $(3.1)$ is such that $(-D \operatorname{grad} \phi, \phi) \in$ $\mathbf{H}(\operatorname{div}, \mathcal{R}) \times H_{0}^{1}(\mathcal{R})$ is a solution to (4.1) with the same data.

In practice, writing the diffusion equation in its mixed form allows to compute precisely both the solution and its gradient: it avoids the propagation of the numerical error from the solution to its gradient. In order to obtain the variational formulation for the mixed problem (4.1), we consider any test functions $\mathbf{q} \in \mathbf{H}(\operatorname{div}, \mathcal{R})$ and $\psi \in L^{2}(\mathcal{R})$, we multiply the first equation of (4.1) by $\mathbf{q}$, the second equation of (4.1) by $\psi \in L^{2}(\mathcal{R})$, and 
we integrate over $\mathcal{R}$. We sum the contributions to reach:

$$
\int_{\mathcal{R}}\left(-D^{-1} \mathbf{p} \cdot \mathbf{q}-\operatorname{grad} \phi \cdot \mathbf{q}+\psi \operatorname{div} \mathbf{p}+\Sigma_{a} \phi \psi\right)=\int_{\mathcal{R}} S_{f} \psi
$$

We recall that $\phi \in H_{0}^{1}(\mathcal{R})$. One can integrate by parts to remove the first order derivatives of $\phi$ :

$$
-\int_{\mathcal{R}} \operatorname{grad} \phi \cdot \mathbf{q}=\int_{\mathcal{R}} \phi \operatorname{div} \mathbf{q}
$$

Hence, the regularity requirement on the solution can be lowered to $\phi \in L^{2}(\mathcal{R})$, and we find that the solution to (4.1) also solves:

Find $(\mathbf{p}, \phi) \in \mathbf{X}$, such that $\forall(\mathbf{q}, \psi) \in \mathbf{X}$ :

$$
\int_{\mathcal{R}}\left(-D^{-1} \mathbf{p} \cdot \mathbf{q}+\phi \operatorname{div} \mathbf{q}+\psi \operatorname{div} \mathbf{p}+\Sigma_{a} \phi \psi\right)=\int_{\mathcal{R}} S_{f} \psi
$$

We define the bilinear forms:

$$
\begin{gathered}
a:\left\{\begin{array}{l}
\mathbf{H}(\operatorname{div}, \mathcal{R}) \times \mathbf{H}(\operatorname{div}, \mathcal{R}) \rightarrow \mathbb{R} \\
(\mathbf{p}, \mathbf{q}) \mapsto \int_{\mathcal{R}}-D^{-1} \mathbf{p} \cdot \mathbf{q}
\end{array} ;\right. \\
b:\left\{\begin{array}{l}
\mathbf{H}(\operatorname{div}, \mathcal{R}) \times L^{2}(\mathcal{R}) \rightarrow \mathbb{R} \\
(\mathbf{q}, \psi) \mapsto \int_{\mathcal{R}} \psi \operatorname{div} \mathbf{q}
\end{array} ;\right. \\
t:\left\{\begin{array}{l}
L^{2}(\mathcal{R}) \times L^{2}(\mathcal{R}) \rightarrow \mathbb{R} \\
(\phi, \psi) \mapsto \int_{\mathcal{R}} \Sigma_{a} \phi \psi
\end{array}\right.
\end{gathered}
$$

and:

$$
c:\left\{\begin{array}{l}
\mathbf{X} \times \mathbf{X} \rightarrow \mathbb{R} \\
(\zeta, \xi) \mapsto a(\mathbf{p}, \mathbf{q})+b(\mathbf{q}, \phi)+b(\mathbf{p}, \psi)+t(\phi, \psi)
\end{array} .\right.
$$

Remark 4.2. The form $c(\cdot, \cdot)$ is symmetric as soon as the tensor field $D$ is symmetric.

We consider the linear form:

$$
f:\left\{\begin{array}{l}
\mathbf{X} \rightarrow \mathbb{R} \\
\xi \mapsto \int_{\mathcal{R}} S_{f} \psi
\end{array}\right.
$$

We may rewrite the variational formulation (4.3) as:

Find $\zeta \in \mathbf{X}$ such that $\forall \xi \in \mathbf{X}$ :

$$
c(\zeta, \xi)=f(\xi) .
$$


Theorem 4.3. The solution $\zeta=(\mathbf{p}, \phi)$ to (4.9) satisfies (4.1). Hence, problems (4.9) and (4.1) are equivalent.

\subsection{Well-posedness of the mixed formulation}

We now recall how to obtain the well-posedness of (4.9) by proving in particular an inf-sup condition.

Theorem 4.4. Let $D$ and $\Sigma_{a}$ satisfy (3.3). Then, there exists a unique solution $\zeta \in \mathbf{X}$ to the mixed variational formulation (4.9).

Proof. Since the form $c(\cdot, \cdot)$ is symmetric, the inf-sup condition yields the claim. This condition writes:

$$
\exists \eta>0, \quad \inf _{\zeta \in \mathbf{X}} \sup _{\xi \in \mathbf{X}} \frac{c(\zeta, \xi)}{\|\zeta\|_{\mathbf{X}}\|\xi\|_{\mathbf{X}}} \geq \eta
$$

To achieve (4.10), a possible choice is:

$$
\left\{\begin{array}{l}
\mathbf{q}=-\mathbf{p} \in \mathbf{H}(\operatorname{div}, \mathcal{R}), \\
\psi=\frac{1}{2} \phi+\frac{1}{2}\left(\Sigma_{a}\right)^{-1} \operatorname{div} \mathbf{p} \in L^{2}(\mathcal{R}) .
\end{array}\right.
$$

It holds $\|\zeta\|_{\mathbf{X}} \geq v\|\xi\|_{\mathbf{X}}$, with $\left.v:=\left(1+\frac{1}{4}\left(\left(\Sigma_{a}\right)_{*}\right)^{-2}\right)\right)^{-1 / 2}$. The bound on $c$ reads:

$$
c(\zeta, \xi) \geq \gamma v\|\zeta\|_{\mathbf{x}}\|\xi\|_{\mathbf{x}}
$$

with $\gamma:=\min \left(\left(D^{*}\right)^{-1}, \frac{1}{2}\left(\Sigma_{a}\right)_{*}, \frac{1}{2}\left(\left(\Sigma_{a}\right)^{*}\right)^{-1}\right)$.

\subsection{Discretization}

We study conforming discretizations of the variational formulation (4.9). To fix ideas, we use a family of triangulations, indexed by a parameter $h$, which is classically chosen as the largest diameter of elements of the triangulation. We introduce discrete, finite-dimensional, spaces indexed by $h$ as follows:

$$
\mathbf{Q}_{h} \subset \mathbf{H}(\operatorname{div}, \mathcal{R}) \text {, and } L_{h} \subset L^{2}(\mathcal{R}) .
$$

For approximation purposes, and following Definition 2.14 in [17], we assume that $\left(\mathbf{Q}_{h}\right)_{h}$, resp. $\left(L_{h}\right)_{h}$ have the approximability property in the sense that

$$
\begin{aligned}
& \forall \mathbf{q} \in \mathbf{H}(\operatorname{div}, \mathcal{R}), \lim _{h \rightarrow 0}\left(\inf _{\mathbf{q}_{h} \in \mathbf{Q}_{h}}\left\|\mathbf{q}-\mathbf{q}_{h}\right\|_{\mathbf{H}(\operatorname{div}, \mathcal{R})}\right)=0, \\
& \forall \psi \in L^{2}(\mathcal{R}), \lim _{h \rightarrow 0}\left(\inf _{\psi_{h} \in L_{h}}\left\|\psi-\psi_{h}\right\|_{0, \mathcal{R}}\right)=0
\end{aligned}
$$

and also that $L_{h}$ includes the subspace $L_{h}^{0}$ of piecewise constant fields on the triangulation.

We impose: $\operatorname{div} \mathbf{Q}_{h} \subset L_{h}$.

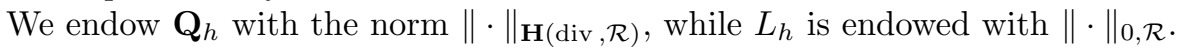

We finally define:

$$
\mathbf{X}_{h}=\left\{\xi_{h}:=\left(\mathbf{q}_{h}, \psi_{h}\right) \in \mathbf{Q}_{h} \times L_{h}\right\}, \text { endowed with }\|\cdot\|_{\mathbf{X}} .
$$

The conforming discretization of the variational formulation (4.9) reads: 
Find $\left(\mathbf{p}_{h}, \phi_{h}\right) \in \mathbf{X}_{h}$, such that $\forall\left(\mathbf{q}_{h}, \psi_{h}\right) \in \mathbf{X}_{h}$ :

$$
a\left(\mathbf{p}_{h}, \mathbf{q}_{h}\right)+b\left(\mathbf{q}_{h}, \phi_{h}\right)+b\left(\mathbf{p}_{h}, \psi_{h}\right)+t\left(\phi_{h}, \psi_{h}\right)=\left(S_{f}, \psi_{h}\right)_{0, \mathcal{R}}
$$

Or equivalently:

$$
\text { Find } \zeta_{h} \in \mathbf{X}_{h} \text { such that } \forall \xi_{h} \in \mathbf{X}_{h}, c\left(\zeta_{h}, \xi_{h}\right)=f\left(\xi_{h}\right) \text {. }
$$

For later use, we denote $\pi^{0}$ the $L^{2}(\mathcal{R})$ orthogonal projector on its subspace $L_{h}^{0}$. By construction, it holds range $\left(\pi^{0}\right)=L_{h}^{0}$ where $\pi^{0}$ is defined by:

$$
\forall \psi \in L^{2}(\mathcal{R}), \forall \psi_{h} \in L_{h}^{0}, \quad\left(\pi^{0} \psi-\psi, \psi_{h}\right)_{0, \mathcal{R}}=0 .
$$

According to Proposition 1.135 of [17]:

$$
\begin{aligned}
& \forall z \in L^{2}(\mathcal{R}), \quad\left\|z-\pi^{0} z\right\|_{0, \mathcal{R}} \lesssim\|z\|_{0, \mathcal{R}} \\
& \forall z \in \mathcal{P} H^{1}(\mathcal{R}), \quad\left\|z-\pi^{0} z\right\|_{0, \mathcal{R}} \lesssim h\|z\|_{\mathcal{P} H^{1}(\mathcal{R})} \\
& \forall z \in \mathcal{P} W^{1, \infty}(\mathcal{R}), \quad\left\|z-\pi^{0} z\right\|_{\infty, \mathcal{R}} \lesssim h\|z\|_{\mathcal{P} W^{1, \infty}(\mathcal{R})}
\end{aligned}
$$

For the last two inequalities, the result holds provided that the triangulations are conforming with respect to the partition, namely for all triangulations, for all elements $K$ of a triangulation, it holds that there exists $1 \leq i \leq N$ such that $K \subset \overline{\mathcal{R}_{i}}$. Similar results hold on subsets of $\mathcal{R}$.

\subsection{Discrete inf-sup condition}

The discrete inf-sup condition to be found writes:

$$
\exists \eta_{h}>0, \quad \inf _{\zeta_{h} \in \mathbf{X}_{h}} \sup _{\xi_{h} \in \mathbf{X}_{h}} \frac{c\left(\zeta_{h}, \xi_{h}\right)}{\left\|\zeta_{h}\right\|_{\mathbf{X}}\left\|\xi_{h}\right\|_{\mathbf{X}}} \geq \eta_{h}
$$

Once (4.16) is achieved, one obtains existence and uniqueness of the discrete solution $\zeta_{h}$, hence the corresponding linear system is well-posed. More generally, our aim is to obtain that $\left(\eta_{h}\right)_{h}$ is uniformly bounded away from 0 . In this sense, one has at hand a uniform discrete inf-sup condition (udisc), from which the error analysis can classically be derived.

Theorem 4.5. Let $D$, resp. $\Sigma_{a} \in \mathcal{P} W^{1, \infty}(\mathcal{R})$, satisfy (3.3). The discrete inf-sup condition (4.16) is fulfilled. Moreover, it is a uniform discrete inf-sup condition.

Proof. In order to prove the discrete inf-sup condition, we use the same method as for the continuous infsup condition ( $c f$. proof of Thm. 4.4). One can remark that if $\Sigma_{a}$ is piecewise-constant, $\frac{1}{2}\left(\Sigma_{a}\right)^{-1} \operatorname{div} \mathbf{p}_{h}$ is automatically in $L_{h}$.

Otherwise, we project $\left(\Sigma_{a}\right)^{-1}$ on the piecewise-constant functions. One modifies (4.11) by choosing:

$$
\left\{\begin{array}{l}
\mathbf{q}_{h}=-\mathbf{p}_{h} \in \mathbf{Q}_{h}, \\
\psi_{h}=\frac{1}{2} \phi_{h}+\frac{1}{2} \pi^{0}\left(\left(\Sigma_{a}\right)^{-1}\right) \operatorname{div} \mathbf{p}_{h} \in L_{h}
\end{array}\right.
$$

Using (4.15) with $z=\left(\Sigma_{a}\right)^{-1}$ yields $\left\|\left(\Sigma_{a}\right)^{-1}-\pi^{0}\left(\left(\Sigma_{a}\right)^{-1}\right)\right\|_{\infty, \mathcal{R}} \lesssim h$, which allows us to derive again a udisc in this more general case. 


\subsection{Numerical analysis of the source problem}

We consider the neutron diffusion equation assuming that $D$, resp. $\Sigma_{a} \in \mathcal{P} W^{1, \infty}(\mathcal{R})$, satisfy (3.3). Under the assumptions of Section 4.3, it follows from the previous study that $\lim _{h \rightarrow 0}\left\|\zeta-\zeta_{h}\right\|_{\mathbf{x}}=0$. We find below a sharper bound of the error $\left\|\zeta-\zeta_{h}\right\|_{\mathbf{X}}$ by using Proposition 3.1. In order to obtain optimal a priori error estimates, we must know the regularity of the solution to problem (3.1). Since we have assumed that the source term $S_{f}$ belongs to $L^{2}(\mathcal{R})$, we already know that $\|\phi\|_{1, \mathcal{R}} \lesssim\left\|S_{f}\right\|_{0, \mathcal{R}}$. Moreover, under the assumptions of Proposition 3.1, the solution $\phi$ has some extra regularity, and the low-regularity case corresponds to $r_{\max }<1 / 2$ there. This is the case that we are focusing on now. In this setting, the field $\mathbf{p}:=-D \operatorname{grad} \phi$ automatically belongs to $\mathcal{P} \mathbf{H}^{r}(\mathcal{R})$, for $0 \leq r<r_{\max }$. We suppose in addition that

$$
\exists \mu \in] 0, r_{\max }\left[, \quad S_{f} \in \mathcal{P} H^{\mu}(\mathcal{R}) .\right.
$$

Then we have $\operatorname{div} \mathbf{p} \in \mathcal{P} H^{\mu}(\mathcal{R})$ (recall $\mathcal{P} H^{\mu}(\mathcal{R})=H^{\mu}(\mathcal{R})$ for $\mu<1 / 2$ ). We will use this hypothesis on $S_{f}$ to carry on the calculations of the error estimates.

We recall below the definition of the Raviart-Thomas-Nédélec (or RTN) finite element [26, 28]. Let $\left(K_{\ell}\right)_{1 \leq \ell \leq L}$ be a conforming mesh, or triangulation, of $\overline{\mathcal{R}}$ made of parallelepipeds (a mesh, or triangulation, is said to be conforming if in every $K_{\ell}, D$ and $\Sigma_{a}$ are smooth). Let $P\left(K_{\ell}\right)$ be the set of polynomials defined over $K_{\ell}$. For integer values $l, m, p \geq 0$, we consider the following subspace of $P\left(K_{\ell}\right)$ :

$$
Q_{l, m, p}\left(K_{\ell}\right)=\left\{q(x, y, z) \in P\left(K_{\ell}\right) \mid q(x, y, z)=\sum_{e, j, k=0}^{l, m, p} a_{e, j, k} x^{e} y^{j} z^{k}, a_{e, j, k} \in \mathbb{R}\right\}
$$

For integer $k \geq 0$, let us set $k^{\prime}=k+1$ and introduce the vector polynomial space:

$$
\mathbf{D}_{k}\left(K_{\ell}\right)=\left[Q_{k^{\prime}, k, k}\left(K_{\ell}\right) \times \mathbf{0} \times \mathbf{0}\right] \oplus\left[\mathbf{0} \times Q_{k, k^{\prime}, k}\left(K_{\ell}\right) \times \mathbf{0}\right] \oplus\left[\mathbf{0} \times \mathbf{0} \times Q_{k, k, k^{\prime}}\left(K_{\ell}\right)\right] .
$$

We can now define the $\operatorname{RTN}_{[k]}$ finite element subspace of $\mathbf{H}(\operatorname{div}, \mathcal{R}) \times L^{2}(\mathcal{R})$ :

$$
\begin{aligned}
\mathbf{Q}_{h}^{k} & =\left\{\mathbf{q} \in \mathbf{H}(\operatorname{div}, \mathcal{R}) \mid \forall \ell \in\{1, \ldots, L\}, \mathbf{q}_{\mid K_{\ell}} \in \mathbf{D}_{k}\left(K_{\ell}\right)\right\} \\
L_{h}^{k} & =\left\{\psi \in L^{2}(\mathcal{R}) \mid \forall \ell \in\{1, \ldots, L\}, \psi_{\mid K_{\ell}} \in Q_{k, k, k}\left(K_{\ell}\right)\right\}
\end{aligned}
$$

As required, it holds $\operatorname{div} \mathbf{Q}_{h}^{k} \subset L_{h}^{k}$ and $L_{h}^{0} \subset L_{h}^{k}$. We recall that for any $\mathbf{q}$ in $\mathbf{H}(\operatorname{div}, \mathcal{R})$, its $\mathrm{RTN}_{[k]}$-interpolant $\mathbf{q}_{R}^{k} \in \mathbf{Q}_{h}^{k}$ satisfies:

$$
\forall \psi_{h} \in L_{h}^{k}, b\left(\mathbf{q}-\mathbf{q}_{R}^{k}, \psi_{h}\right)=0
$$

In addition thanks to the commuting diagram property, $c f$. Section 2.5.2 of [7], it holds

$$
\forall \mathbf{q} \in \mathbf{H}(\operatorname{div}, \mathcal{R}), \operatorname{div} \mathbf{q}_{R}^{0}=\pi^{0}(\operatorname{div} \mathbf{q})
$$

Let $\mathbf{q} \in \mathbf{H}^{r}(\mathcal{R})$, such that $\operatorname{div} \mathbf{q} \in H^{s}(\mathcal{R}), 0<r, s<r_{\max }$. According to Lemma 3.3 of [3]:

$$
\begin{aligned}
\left\|\mathbf{q}-\mathbf{q}_{R}^{0}\right\|_{0, \mathcal{R}} & \lesssim\left(h^{r}|\mathbf{q}|_{r, \mathcal{R}}+h\|\operatorname{div} \mathbf{q}\|_{0, \mathcal{R}}\right), \\
\left\|\operatorname{div}\left(\mathbf{q}-\mathbf{q}_{R}^{0}\right)\right\|_{0, \mathcal{R}} & \lesssim h^{s}|\operatorname{div} \mathbf{q}|_{s, \mathcal{R}} .
\end{aligned}
$$

Similar results hold on subsets of $\mathcal{R}$, provided the discretizations are conforming. 
Remark 4.6. If one chooses another discretization, all results presented hereafter hold provided the estimates (4.20) remain true. For instance, for the $\mathrm{RTN}_{[k]}$ finite element defined on tetrahedral triangulations of $\overline{\mathcal{R}}, c f$. Section 2.3.1 of [7]. To prove (4.20) in this case, one has simply to apply the results of Section 3.2 from [3]. On the other hand, provided that the field $\mathbf{q}$ and its divergence are "smooth" in the sense that they belong to $\mathcal{P} H^{m+1}(\mathcal{R})$ for some integer $m \geq 0$, using the $\operatorname{RTN}_{[m]}$ finite element one can recover interpolation estimates in $O\left(h^{m+1}\right)$, cf. Section 2.5.5 in [7]. For meshes made of affine elements such as tetrahedra or parallelepipeds, the approximation estimate (4.20-top) does not require the term with the divergence (see, e.g. [7], Sect. 2.5.1).

\subsubsection{A priori error estimates}

Since we focus on the low-regularity case, we choose the $\mathrm{RTN}_{[0]}$ finite element, i.e. $\mathbf{X}_{h}=\mathbf{Q}_{h}^{0} \times L_{h}^{0}$. If the solution is "smooth", one can increase the order of the RTN finite element. This will be used in particular in Section 4.6.2 for the study of the error on the eigenvalues. According to first Strang's Lemma [17] and because $\left(1+\|c\|\left(\eta_{h}\right)^{-1}\right) \lesssim 1$, the error reads:

$$
\left\|\zeta-\zeta_{h}\right\|_{\mathbf{X}} \lesssim \inf _{\xi_{h} \in \mathbf{X}_{h}}\left\|\zeta-\xi_{h}\right\|_{\mathbf{X}}
$$

Theorem 4.7. Under the assumptions of Proposition 3.1, it holds, with $r_{\max }<1 / 2$ :

$$
\begin{aligned}
& \forall \mu \in] 0, r_{\max }\left[, \quad \forall S_{f} \in H^{\mu}(\mathcal{R}),\right. \\
& \left\|\mathbf{p}-\mathbf{p}_{h}\right\|_{\mathbf{H}(\operatorname{div}, \mathcal{R})}+\left\|\phi-\phi_{h}\right\|_{0, \mathcal{R}} \lesssim h^{\mu}\left\|S_{f}\right\|_{\mu, \mathcal{R}} .
\end{aligned}
$$

Remark 4.8. In particular, for "smooth data" $S_{f}$, i.e. $S_{f} \in H^{r_{\max }}(\mathcal{R})$, one expects a convergence rate at least in $h^{r_{\max }-\eta}$ for $\eta>0$ arbitrary small: by a slight abuse of notation there and in the sequel, we shall write $h^{r_{\max }}$. Also, the previous analysis can be extended to the case where $r_{\max }$ is in $[1 / 2,1]$ and $\mu<r_{\max }$ (or $\mu \leq 1$ if $\left.r_{\max }=1\right)$. Furthermore, for a "smooth" solution, one may recover a convergence rate like $O\left(h^{m+1}\right)$ for an $\operatorname{RTN}_{[m]}$ discretization of order $m \geq 0$.

Proof. Choosing $\xi_{h}=\left(\mathbf{p}_{R}^{0}, \pi^{0} \phi\right) \in \mathbf{X}_{h}$, then thanks to the a priori estimates (4.15) and (4.20), it follows that:

$$
\begin{aligned}
\left\|\zeta-\xi_{h}\right\|_{\mathbf{X}}^{2} & =\left\|\mathbf{p}-\mathbf{p}_{R}^{0}\right\|_{\mathbf{H}(\operatorname{div}, \mathcal{R})}^{2}+\left\|\phi-\pi^{0} \phi\right\|_{0, \mathcal{R}}^{2} \\
& \lesssim h^{2 \mu}\left(|\mathbf{p}|_{\mu, \mathcal{R}}^{2}+\|\operatorname{div} \mathbf{p}\|_{\mu, \mathcal{R}}^{2}\right)+h^{2}\|\phi\|_{1, \mathcal{R}}^{2} \\
& \lesssim h^{2 \mu}\left\|S_{f}\right\|_{\mu, \mathcal{R}}^{2} .
\end{aligned}
$$

\subsubsection{Aubin-Nitsche-type estimates}

To derive improved estimates on the error $\left\|\phi-\phi_{h}\right\|_{0, \mathcal{R}}$ in $\mathbf{X}_{h}=\mathbf{Q}_{h}^{0} \times L_{h}^{0}$, we shall rely on the illuminating work of Falk-Osborn [18]. Interestingly, one can obtain an improvement of the convergence rate, contrary to the case where the solution is "smooth". From the previous analysis, for all $\mu<r_{\max }$, we already have the estimate (4.22).

Lemma 4.9. Let $(\mathbf{p}, \phi)$ (resp. $\left.\left(\mathbf{p}_{h}, \phi_{h}\right)\right)$ the solution of continuous (resp. discrete) variational problem (4.3) (resp. (4.13)). For all $\left(\mathbf{q}_{h}, \psi_{h}\right)$ in $\mathbf{X}_{h}$, it holds:

$$
\begin{aligned}
& a\left(\mathbf{p}-\mathbf{p}_{h}, \mathbf{q}_{h}\right)+b\left(\mathbf{q}_{h}, \phi-\phi_{h}\right)=0, \\
& b\left(\mathbf{p}-\mathbf{p}_{h}, \psi_{h}\right)+t\left(\phi-\phi_{h}, \psi_{h}\right)=0 .
\end{aligned}
$$


Proof. Let $\left(\mathbf{q}_{h}, \psi_{h}\right)$ be in $\mathbf{X}_{h}$. The subtraction of (4.3) from (4.13), with $(\mathbf{q}, \psi)=\left(\mathbf{q}_{h}, \psi_{h}\right)$ in the former, gives

$$
a\left(\mathbf{p}-\mathbf{p}_{h}, \mathbf{q}_{h}\right)+b\left(\mathbf{q}_{h}, \phi-\phi_{h}\right)+b\left(\mathbf{p}-\mathbf{p}_{h}, \psi_{h}\right)+t\left(\phi-\phi_{h}, \psi_{h}\right)=0 .
$$

We obtain the first equality (4.23) (resp. the second equality (4.24)) with $\psi_{h}=0$ (resp. $\left.\mathbf{q}_{h}=\mathbf{0}\right)$.

Before improving the estimate, we need to introduce the adjoint problem:

For $d \in L^{2}(\mathcal{R})$, find $\left(\mathbf{y}_{d}, \eta_{d}\right) \in \mathbf{X}$ such that $\forall(\mathbf{q}, \psi) \in \mathbf{X}$ :

$$
a\left(\mathbf{y}_{d}, \mathbf{q}\right)+b\left(\mathbf{q}, \eta_{d}\right)+b\left(\mathbf{y}_{d}, \psi\right)+t\left(\eta_{d}, \psi\right)=(d, \psi)_{0, \mathcal{R}}
$$

Theorem 4.10. Under the assumptions of Proposition 3.1, it holds, with $r_{\max }<1 / 2$ :

$$
\forall \mu \in] 0, r_{\max }\left[, \quad \forall S_{f} \in H^{\mu}(\mathcal{R}), \quad\left\|\phi-\phi_{h}\right\|_{0, \mathcal{R}} \lesssim h^{2 \mu}\left\|S_{f}\right\|_{\mu, \mathcal{R}}\right.
$$

Proof. Adapting the methodology of [18] and by using $\left(0, \phi-\phi_{h}\right)$ as a test function in the adjoint problem (4.25), we remark:

$$
\left\|\phi-\phi_{h}\right\|_{0, \mathcal{R}}=\sup _{d \in L^{2}(\mathcal{R}) \backslash\{0\}} \frac{b\left(\mathbf{y}_{d}, \phi-\phi_{h}\right)+t\left(\eta_{d}, \phi-\phi_{h}\right)}{\|d\|_{0, \mathcal{R}}} .
$$

We now look for an upper bound of the supremum in (4.27). We find that the numerator is successively equal to:

$$
b\left(\mathbf{y}_{d}-\left(\mathbf{y}_{d}\right)_{R}^{0}, \phi-\phi_{h}\right)+b\left(\left(\mathbf{y}_{d}\right)_{R}^{0}, \phi-\phi_{h}\right)+t\left(\eta_{d}, \phi-\phi_{h}\right)
$$

using (4.18), for any $\psi_{h}^{*}, \psi_{h}^{\prime}$ in $L_{h}$ :

$$
b\left(\mathbf{y}_{d}-\left(\mathbf{y}_{d}\right)_{R}^{0}, \phi-\psi_{h}^{*}\right)+b\left(\left(\mathbf{y}_{d}\right)_{R}^{0}, \phi-\phi_{h}\right)+t\left(\eta_{d}-\psi_{h}^{\prime}, \phi-\phi_{h}\right)+t\left(\psi_{h}^{\prime}, \phi-\phi_{h}\right) ;
$$

using (4.23) with $\mathbf{q}_{h}=\left(\mathbf{y}_{d}\right)_{R}^{0}$ :

$$
b\left(\mathbf{y}_{d}-\left(\mathbf{y}_{d}\right)_{R}^{0}, \phi-\psi_{h}^{*}\right)-a\left(\mathbf{p}-\mathbf{p}_{h},\left(\mathbf{y}_{d}\right)_{R}^{0}\right)+t\left(\eta_{d}-\psi_{h}^{\prime}, \phi-\phi_{h}\right)+t\left(\psi_{h}^{\prime}, \phi-\phi_{h}\right) ;
$$

now we use (4.24) with $\psi_{h}=\psi_{h}^{\prime}$ :

$$
b\left(\mathbf{y}_{d}-\left(\mathbf{y}_{d}\right)_{R}^{0}, \phi-\psi_{h}^{*}\right)-a\left(\mathbf{p}-\mathbf{p}_{h},\left(\mathbf{y}_{d}\right)_{R}^{0}\right)+t\left(\eta_{d}-\psi_{h}^{\prime}, \phi-\phi_{h}\right)-b\left(\mathbf{p}-\mathbf{p}_{h}, \psi_{h}^{\prime}\right) ;
$$

we add (4.25) with $\left(\mathbf{p}-\mathbf{p}_{h}, 0\right)$ as a test function:

$$
b\left(\mathbf{y}_{d}-\left(\mathbf{y}_{d}\right)_{R}^{0}, \phi-\psi_{h}^{*}\right)+a\left(\mathbf{p}-\mathbf{p}_{h}, \mathbf{y}_{d}-\left(\mathbf{y}_{d}\right)_{R}^{0}\right)+t\left(\eta_{d}-\psi_{h}^{\prime}, \phi-\phi_{h}\right)+b\left(\mathbf{p}-\mathbf{p}_{h}, \eta_{d}-\psi_{h}^{\prime}\right) .
$$

All terms ${ }^{1}$ in the previous relation can be bounded with an $h$-dependent term:

$$
\begin{aligned}
\inf _{\psi_{h}^{*} \in L_{h}}\left|b\left(\mathbf{y}_{d}-\left(\mathbf{y}_{d}\right)_{R}^{0}, \phi-\psi_{h}^{*}\right)\right| & \lesssim\left\|\operatorname{div}\left(\mathbf{y}_{d}-\left(\mathbf{y}_{d}\right)_{R}^{0}\right)\right\|_{0, \mathcal{R}} \inf _{\psi_{h}^{*} \in L_{h}}\left\|\phi-\psi_{h}^{*}\right\|_{0, \mathcal{R}} \\
& \lesssim\left\|\operatorname{div} \mathbf{y}_{d}\right\|_{0, \mathcal{R}} h\|\phi\|_{1, \mathcal{R}} \\
& \lesssim h\left\|S_{f}\right\|_{\mu, \mathcal{R}}\|d\|_{0, \mathcal{R}} ;
\end{aligned}
$$

\footnotetext{
${ }^{\mathrm{I}}$ In particular, $\left\|\operatorname{div}\left(\mathbf{y}_{d}-\left(\mathbf{y}_{d}\right)_{R}^{0}\right)\right\|_{0, \mathcal{R}} \lesssim\left\|\operatorname{div} \mathbf{y}_{d}\right\|_{0, \mathcal{R}}$ according to (4.15) and (4.19).
} 


$$
\begin{aligned}
\left|a\left(\mathbf{p}-\mathbf{p}_{h}, \mathbf{y}_{d}-\left(\mathbf{y}_{d}\right)_{R}^{0}\right)\right| & \lesssim\left\|\mathbf{p}-\mathbf{p}_{h}\right\|_{0, \mathcal{R}}\left\|\mathbf{y}_{d}-\left(\mathbf{y}_{d}\right)_{R}^{0}\right\|_{0, \mathcal{R}} \\
& \lesssim h^{\mu}\left\|S_{f}\right\|_{\mu, \mathcal{R}}\left(h^{\mu}\left|\mathbf{y}_{d}\right|_{\mu, \mathcal{R}}+h\left\|\operatorname{div} \mathbf{y}_{d}\right\|_{0, \mathcal{R}}\right) \\
& \lesssim h^{2 \mu}\left\|S_{f}\right\|_{\mu, \mathcal{R}}\|d\|_{0, \mathcal{R}}
\end{aligned}
$$

The last two terms in (4.28) are considered together.

$$
\begin{aligned}
& \inf _{\psi_{h}^{\prime} \in L_{h}}\left|b\left(\mathbf{p}-\mathbf{p}_{h}, \eta_{d}-\psi_{h}^{\prime}\right)+t\left(\phi-\phi_{h}, \eta_{d}-\psi_{h}^{\prime}\right)\right| \\
& \lesssim\left(\left\|\operatorname{div}\left(\mathbf{p}-\mathbf{p}_{h}\right)\right\|_{0, \mathcal{R}}+\left\|\phi-\phi_{h}\right\|_{0, \mathcal{R}}\right) \inf _{\psi_{h}^{\prime} \in L_{h}}\left\|\eta_{d}-\psi_{h}^{\prime}\right\|_{0, \mathcal{R}} \\
& \lesssim h^{\mu}\left\|S_{f}\right\|_{\mu, \mathcal{R}} \inf _{\psi_{h}^{\prime} \in L_{h}}\left\|\eta_{d}-\psi_{h}^{\prime}\right\|_{0, \mathcal{R}} \\
& \lesssim h^{\mu}\left\|S_{f}\right\|_{\mu, \mathcal{R}} h\left\|\eta_{d}\right\|_{1, \mathcal{R}} \lesssim h^{\mu+1}\left\|S_{f}\right\|_{\mu, \mathcal{R}}\|d\|_{0, \mathcal{R}} .
\end{aligned}
$$

Thus, for low-regularity solutions $(\mu<1 / 2)$, we conclude that it holds:

$$
\left\|\phi-\phi_{h}\right\|_{0, \mathcal{R}} \lesssim \max \left(h, h^{2 \mu}, h^{\mu+1}\right)\left\|S_{f}\right\|_{\mu, \mathcal{R}} \approx h^{2 \mu}\left\|S_{f}\right\|_{\mu, \mathcal{R}}
$$

Corollary 4.11. In the case of "smooth data" $S_{f}$, i.e. $S_{f} \in H^{r_{\max }}(\mathcal{R})$, the error estimate gives:

$$
\left\|\phi-\phi_{h}\right\|_{0, \mathcal{R}} \lesssim h^{2 r_{\max }}\left\|S_{f}\right\|_{r_{\max }, \mathcal{R}}
$$

\subsection{Numerical analysis of the generalized eigenvalue problem}

Let us focus on the approximation of the generalized eigenvalue problem (3.2) in our low-regularity setting, under the assumptions of Proposition 3.1, supplemented with $\underline{\nu \Sigma}_{f} \in \mathcal{P} W^{1, \infty}(\mathcal{R})$.

Let $0 \leq \mu<r_{\max }$ be given, we introduce an operator $B_{\mu}$ associated to the source problem (4.3): given $f \in H^{\mu}(\mathcal{R})$, we call $B_{\mu} f=\phi \in H^{1}(\mathcal{R})$ the second component of the couple (p, $\left.\phi\right)$ that solves (4.3) with source $S_{f}=\underline{\nu \Sigma_{f}} f$. Since $\underline{\nu \Sigma_{f}}$ belongs to $\mathcal{P} W^{1, \infty}(\mathcal{R})$, it holds $\left\|S_{f}\right\|_{\mu, \mathcal{R}} \lesssim\|f\|_{\mu, \mathcal{R}}$ because $\mu<1 / 2$. Hence, $B_{\mu}$ is a bounded operator from $H^{\mu}(\mathcal{R})$ to itself:

$$
\left\|B_{\mu} f\right\|_{\mu, \mathcal{R}} \lesssim\left\|B_{\mu} f\right\|_{1, \mathcal{R}}=\|\phi\|_{1, \mathcal{R}} \lesssim\left\|S_{f}\right\|_{0, \mathcal{R}} \lesssim\left\|S_{f}\right\|_{\mu, \mathcal{R}} \lesssim\|f\|_{\mu, \mathcal{R}}
$$

we write $B_{\mu} \in \mathcal{L}\left(H^{\mu}(\mathcal{R})\right)$ for short. In addition, since the second component of the solution actually belongs to $H^{1}(\mathcal{R})$ with continuous dependence $\left(\|\phi\|_{1, \mathcal{R}} \lesssim\|f\|_{\mu, \mathcal{R}}\right)$, it follows that $B_{\mu}$ is a compact operator. Denote by $\sigma\left(B_{\mu}\right)$ its spectrum. By construction, $\lambda^{-1} \in \sigma\left(B_{\mu}\right)$ if, and only if, $\lambda$ is an eigenvalue of (3.2).

Finally, we consider the discrete operator $B_{\mu}^{h}$ associated to the discrete source problem (4.13): given $f \in$ $H^{\mu}(\mathcal{R})$, we call $B_{\mu}^{h} f$ the second component of the couple $\left(\mathbf{p}_{h}, \phi_{h}\right)$ that solves (4.13) with source $S_{f}=\underline{\nu \Sigma_{f} f}$.

Under the assumptions of Section 4.3 and as noted at the beginning of Section 4.5, it holds $\lim _{h \rightarrow 0} \| B_{0} f-$ $B_{0}^{h} f \|_{0, \mathcal{R}}=0$ for all $f \in L^{2}(\mathcal{R})$. This property is the so-called pointwise convergence. However, for a mixed formulation, the fact that the family $\left(B_{0}^{h}\right)_{h}$ converges pointwise towards the compact operator $B_{0}$ is not sufficient to guarantee that the family $\left(B_{0}^{h}\right)_{h}$ converges in operator norm towards $B_{0}$.

\subsubsection{Convergence in operator norm}

On the other hand, according to [27], proving that $\lim _{h \rightarrow 0}\left\|B_{\mu}-B_{\mu}^{h}\right\|_{\mathcal{L}\left(H^{\mu}(\mathcal{R})\right)}=0$ for discrete approximants $\left(B_{\mu}^{h}\right)_{h}$ is a sufficient condition to obtain convergence of the eigenvalues. In order to ensure the convergence in operator norm of the family $\left(B_{\mu}^{h}\right)_{h}$ towards the compact operator $B_{\mu}$, we need a technical assumption on the triangulations. 
Definition 4.12. A family of triangulations $\left(\mathcal{T}_{h}\right)_{h}$ is regular ${ }^{+}$if it satisfies:

$$
\exists \theta>0, \forall h, h^{2-\theta} \lesssim \min _{K \in \mathcal{T}_{h}} \operatorname{diam}(K) .
$$

In particular, a quasi-uniform family of triangulations is regular ${ }^{+}$(take $\theta=1$ in (4.29)). For a regular family, one has the following inverse inequality, whose proof is given in Appendix A.

Lemma 4.13. Let $\mu \in\left[0,1 / 2\left[\right.\right.$. For a regular $^{+}$family of triangulations, it holds:

$$
\forall h, \forall \psi_{h} \in L_{h}^{k},\left\|\psi_{h}\right\|_{\mu, \mathcal{R}} \lesssim h^{-2 \mu+\theta \mu}\left\|\psi_{h}\right\|_{0, \mathcal{R}}
$$

Theorem 4.14. Under the assumptions of Proposition 3.1 with $r_{\max }<1 / 2$ plus $\underline{\nu}_{f} \in \mathcal{P} W^{1, \infty}(\mathcal{R})$, let $\mu \in$ $\left[0, r_{\max }\left[\right.\right.$. Provided that the family of triangulations is regular ${ }^{+}$, one has:

$$
\left\|B_{\mu}-B_{\mu}^{h}\right\|_{\mathcal{L}\left(H^{\mu}(\mathcal{R})\right)} \lesssim h^{\theta \mu}
$$

Proof. According to (4.26), we know that

$$
\left\|\left(B_{\mu}-B_{\mu}^{h}\right) f\right\|_{0, \mathcal{R}} \lesssim h^{2 \mu}\|f\|_{\mu, \mathcal{R}}
$$

It remains to estimate $\left\|\left(B_{\mu}-B_{\mu}^{h}\right) f\right\|_{\mu, \mathcal{R}}$ : for that, we use the triangle inequality

$$
\left\|\left(B_{\mu}-B_{\mu}^{h}\right) f\right\|_{\mu, \mathcal{R}} \leq\left\|B_{\mu} f-\pi^{0}\left(B_{\mu} f\right)\right\|_{\mu, \mathcal{R}}+\left\|\pi^{0}\left(B_{\mu} f\right)-B_{\mu}^{h} f\right\|_{\mu, \mathcal{R}} .
$$

To bound the first term, we have according to Theorem 2.3 in [2] that

$$
\forall \psi \in \mathcal{P} H^{1}(\mathcal{R}),\left\|\psi-\pi^{0} \psi\right\|_{\mu, \mathcal{R}} \lesssim h^{1-\mu}\|\psi\|_{\mathcal{P} H^{1}(\mathcal{R})} .
$$

Applying the result to $\psi=B_{\mu} f$, we find $\left\|B_{\mu} f-\pi^{0}\left(B_{\mu} f\right)\right\|_{\mu, \mathcal{R}} \lesssim h^{1-\mu}\|f\|_{\mu, \mathcal{R}}$.

To bound the second term, we use first the inverse inequality (4.30) on the discrete space $L_{h}^{k}$, valid for a regular $^{+}$family of triangulations. Applying the result to $\psi_{h}=\pi^{0}\left(B_{\mu} f\right)-B_{\mu}^{h} f$ and using again the triangle inequality, we now find that

$$
\begin{aligned}
\left\|\pi^{0}\left(B_{\mu} f\right)-B_{\mu}^{h} f\right\|_{\mu, \mathcal{R}} & \lesssim h^{-2 \mu+\theta \mu}\left\|\pi^{0}\left(B_{\mu} f\right)-B_{\mu}^{h} f\right\|_{0, \mathcal{R}} \\
& \lesssim h^{-2 \mu+\theta \mu}\left(\left\|\pi^{0}\left(B_{\mu} f\right)-B_{\mu} f\right\|_{0, \mathcal{R}}+\left\|B_{\mu} f-B_{\mu}^{h} f\right\|_{0, \mathcal{R}}\right) \\
& \lesssim \max \left(h^{1-2 \mu+\theta \mu}, h^{\theta \mu}\right)\|f\|_{\mu, \mathcal{R}},
\end{aligned}
$$

where we have used (4.15) and (4.32) to derive the final estimate. Since $\mu<1 / 2$, we conclude by aggregating the results that (4.31) holds.

Thanks to [27], convergence of the discrete eigenvalues to the exact ones is guaranteed, and so is the absence of spectral pollution:

- Given any closed, non-empty disk $D \subset \mathbb{C}$ such that $D \cap \sigma\left(B_{\mu}\right)=\emptyset$, there exists $h_{0}>0$ such that, for all $h<h_{0}, D \cap \sigma\left(B_{\mu}^{h}\right)=\emptyset$.

- Given any closed, non-empty disk $D \subset \mathbb{C}$ such that $D \cap \sigma\left(B_{\mu}\right)=\{\lambda\}$, with $\lambda$ of multiplicity $m_{\lambda}$, there exists $h_{0}>0$ such that, for all $h<h_{0}, D \cap \sigma\left(B_{\mu}^{h}\right)$ contains exactly $m_{\lambda}$ discrete eigenvalues. 


\subsubsection{Optimal convergence rate}

Let the assumptions of Theorem 4.14 hold. We determine now the rate of convergence of the eigenvalues in the spirit of [8]. Let $\nu=\lambda^{-1}$ be an eigenvalue of $B_{\mu}$. For simplicity, let us assume that $\nu$ is a simple eigenvalue, and denote by $W$ the associated eigenspace. According to the absence of spectral pollution, for $h$ small enough, the closest discrete eigenvalue, denoted by $\nu_{h}$, is also simple; we denote by $W_{h}$ the associated eigenspace.

Definition 4.15. Let $\omega_{\nu}>0$ be the regularity exponent of the eigenfunction, i.e. either $W \subset \mathcal{P} H^{1+s}(\mathcal{R})$ for $s<\omega_{\nu}$ and $W \not \subset \mathcal{P} H^{1+\omega_{\nu}}(\mathcal{R})$, or $W \subset \mathcal{P} H^{1+\omega_{\nu}}(\mathcal{R})$ and $W \not \subset \mathcal{P} H^{1+s}(\mathcal{R})$ for $s>\omega_{\nu}$. Let $\omega=\min \left(\omega_{\nu}, m+1\right)$, where $m \geq 0$ is the order of the RTN finite element.

Clearly, $\omega_{\nu}$, and as a consequence $\omega$, can be greater than $r_{\max }$. We shall prove that the approximation converges with a rate equal to twice the exponent $\omega$ defined above: this result is stated in Corollary 4.23 at the end of the subsection.

Let $\mu \in\left[0, r_{\max }\left[\right.\right.$ be given. As we defined $B_{\mu}$ (resp. $\left.B_{\mu}^{h}\right)$, we define $A_{\mu}$ (resp. $\left.A_{\mu}^{h}\right)$ : for $f \in H^{\mu}(\mathcal{R})$, we call $A_{\mu} f=\mathbf{p} \in \mathbf{H}(\operatorname{div}, \mathcal{R})\left(\right.$ resp. $\left.A_{\mu}^{h} f=\mathbf{p}_{h} \in \mathbf{Q}_{h}\right)$ the first component of the couple $(\mathbf{p}, \phi)$ (resp. $\left.\left(\mathbf{p}_{h}, \phi_{h}\right)\right)$ that

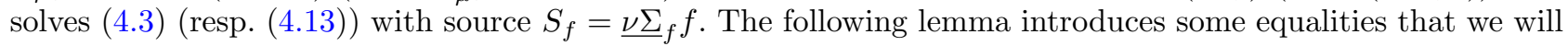
use later on.

Lemma 4.16. Let $\varphi$ and $\varphi^{\prime}$ be given in $W$. Then, it holds:

$$
\begin{aligned}
& \left(\underline{\nu \Sigma}_{f} \varphi,\left(B_{\mu}-B_{\mu}^{h}\right) \varphi^{\prime}\right)_{0, \mathcal{R}}=a\left(A_{\mu} \varphi,\left(A_{\mu}-A_{\mu}^{h}\right) \varphi^{\prime}\right) \\
& \quad+b\left(\left(A_{\mu}-A_{\mu}^{h}\right) \varphi^{\prime}, B_{\mu} \varphi\right)+b\left(A_{\mu} \varphi,\left(B_{\mu}-B_{\mu}^{h}\right) \varphi^{\prime}\right)+t\left(B_{\mu} \varphi,\left(B_{\mu}-B_{\mu}^{h}\right) \varphi^{\prime}\right)
\end{aligned}
$$

and

$$
\begin{aligned}
0= & a\left(A_{\mu}^{h} \varphi,\left(A_{\mu}-A_{\mu}^{h}\right) \varphi^{\prime}\right)+b\left(\left(A_{\mu}-A_{\mu}^{h}\right) \varphi^{\prime}, B_{\mu}^{h} \varphi\right) \\
& +b\left(A_{\mu}^{h} \varphi,\left(B_{\mu}-B_{\mu}^{h}\right) \varphi^{\prime}\right)+t\left(B_{\mu}^{h} \varphi,\left(B_{\mu}-B_{\mu}^{h}\right) \varphi^{\prime}\right) .
\end{aligned}
$$

Proof. The definitions of $A_{\mu}, B_{\mu}$ imply that for all $f \in H^{\mu}(\mathcal{R})$, for all $(\mathbf{q}, \psi) \in \mathbf{X}$ :

$$
\left(\underline{\nu \Sigma_{f}} f, \psi\right)_{0, \mathcal{R}}=a\left(A_{\mu} f, \mathbf{q}\right)+b\left(\mathbf{q}, B_{\mu} f\right)+b\left(A_{\mu} f, \psi\right)+t\left(B_{\mu} f, \psi\right)
$$

whereas the definitions of $A_{\mu}^{h}, B_{\mu}^{h}$ imply that for all $f \in H^{\mu}(\mathcal{R})$, for all $(\mathbf{q}, \psi) \in \mathbf{X}_{h}$ :

$$
\left(\underline{\nu \Sigma}_{f} f, \psi\right)_{0, \mathcal{R}}=a\left(A_{\mu}^{h} f, \mathbf{q}\right)+b\left(\mathbf{q}, B_{\mu}^{h} f\right)+b\left(A_{\mu}^{h} f, \psi\right)+t\left(B_{\mu}^{h} f, \psi\right)
$$

The first equality (4.33) comes from (4.35) with:

$$
f=\varphi ; \mathbf{q}=\left(A_{\mu}-A_{\mu}^{h}\right) \varphi^{\prime} ; \psi=\left(B_{\mu}-B_{\mu}^{h}\right) \varphi^{\prime}
$$

The second one, (4.34), comes from the difference between (4.35) and (4.36) with:

$$
f=\varphi^{\prime} ; \mathbf{q}=A_{\mu}^{h} \varphi ; \psi=B_{\mu}^{h} \varphi
$$

and with the symmetry of $a(\cdot, \cdot)$ and $t(\cdot, \cdot)$. 
We remark that $\varphi \mapsto\|\varphi\|_{W}=\left\|\left(\underline{\nu \Sigma_{f}}\right)^{\frac{1}{2}} \varphi\right\|_{0, \mathcal{R}}$ is a norm over $W{ }^{2}$ and this norm is induced by the inner product

$$
\left(\varphi, \varphi^{\prime}\right)_{W}=\left(\underline{\nu \Sigma}_{f} \varphi, \varphi^{\prime}\right)_{0, \mathcal{R}}
$$

Proposition 4.17. Let $\omega$ be as in Definition 4.15. For every $\varphi$ in $W$, the following inequalities hold:

$$
\begin{aligned}
\left\|\left(B_{\mu}-B_{\mu}^{h}\right) \varphi\right\|_{0, \mathcal{R}} & \lesssim h^{\omega}\|\varphi\|_{W} \\
\left\|\left(A_{\mu}-A_{\mu}^{h}\right) \varphi\right\|_{\mathbf{H}(\operatorname{div}, \mathcal{R})} & \lesssim h^{\omega}\|\varphi\|_{W} .
\end{aligned}
$$

Proof. These two inequalities come from the first Strang's Lemma. The method is the same as for Theorem 4.7 (see Rem. 4.8 for the "smooth" case). Here, we use the equivalence of all norms on $W$ to state the result.

Introducing $\delta\left(Z, Z^{\prime}\right)=\sup _{z \in Z,\|z\|_{0}=1} \inf _{z^{\prime} \in Z^{\prime}}\left\|z-z^{\prime}\right\|_{0, \mathcal{R}}$ for $Z, Z^{\prime}$ closed subspaces of $L^{2}(\mathcal{R})$, the gap between $W$ and $W_{h}$ is defined by:

$$
\hat{\delta}\left(W, W_{h}\right)=\max \left[\delta\left(W, W_{h}\right), \delta\left(W_{h}, W\right)\right] .
$$

It allows us to evaluate the approximation of the continuous eigenfunctions by their discrete counterparts. Classically, this gap can be bounded with the help of Proposition 4.17, following Theorem 1 from [27]:

$$
\hat{\delta}\left(W, W_{h}\right) \lesssim h^{\omega}
$$

Let us now define $E_{h}$ as the projector from $L^{2}(\mathcal{R})$ onto $W_{h}$ such that

$$
\forall \varphi \in L^{2}(\mathcal{R}), \forall \psi_{h} \in W_{h},\left(\underline{\nu \Sigma}_{f}\left(\varphi-E_{h} \varphi\right), \psi_{h}\right)_{0, \mathcal{R}}=0 .
$$

Lemma 4.18. The operators $E_{h}$ and $B_{\mu}^{h}$ commute.

Proof. Let $\varphi \in L^{2}(\mathcal{R})$ be decomposed into $\varphi=E_{h} \varphi+\bar{\varphi}$. By construction $E_{h} \varphi \in W_{h}$, so that $B_{\mu}^{h} E_{h} \varphi \in W_{h}$, hence $E_{h} B_{\mu}^{h} E_{h} \varphi=B_{\mu}^{h} E_{h} \varphi$ because $W_{h}$ is invariant through $E_{h}$. It follows $E_{h} B_{\mu}^{h} \varphi=E_{h} B_{\mu}^{h} E_{h} \varphi+E_{h} B_{\mu}^{h} \bar{\varphi}=$ $B_{\mu}^{h} E_{h} \varphi+E_{h} B_{\mu}^{h} \bar{\varphi}$. This is equivalently expressed as

$$
\left(E_{h} B_{\mu}^{h}-B_{\mu}^{h} E_{h}\right) \varphi=E_{h} B_{\mu}^{h} \bar{\varphi} .
$$

By construction, $\psi_{h}=E_{h} B_{\mu}^{h} \bar{\varphi}$ belongs to $W_{h}$, with squared norm equal to

$$
\left(\underline{\nu \Sigma_{f}} \psi_{h}, \psi_{h}\right)_{0, \mathcal{R}}=\left(\underline{\nu \Sigma}_{f} E_{h} B_{\mu}^{h} \bar{\varphi}, \psi_{h}\right)_{0, \mathcal{R}}=\left(\underline{\nu \Sigma_{f}} B_{\mu}^{h} \bar{\varphi}, \psi_{h}\right)_{0, \mathcal{R}}=\left(\underline{\nu \Sigma_{f}} \bar{\varphi}, B_{\mu}^{h} \psi_{h}\right)_{0, \mathcal{R}}=0 .
$$

The penultimate equality stems from the fact that $c(\cdot, \cdot)$ is symmetric, and the last one comes from the definition of $\bar{\varphi}$ and $E_{h}$.

Let $F_{h}$ be the restriction of $E_{h}$ to $W$. One has the following simple results as a consequence of the gap property.

Lemma 4.19. For $h$ small enough, $F_{h}$ is a bijection from $W$ to $W_{h}$. Moreover

$$
\forall \varphi \in W,\left\|\left(\underline{\nu}_{f}\right)^{\frac{1}{2}}\left(\varphi-F_{h} \varphi\right)\right\|_{0, \mathcal{R}} \lesssim h^{\omega}\|\varphi\|_{W}
$$

\footnotetext{
${ }^{2}$ If $\|\varphi\|_{W}=0$, then $\underline{\nu \Sigma_{f}} \varphi=0$. By definition of $W, \varphi$ is solution of (3.2) with zero right-hand side. Thus, by uniqueness of the solution it follows that $\varphi=0$.
} 
Let $\mathcal{S}_{h}=F_{h}^{-1} E_{h}-I \in \mathcal{L}\left(L^{2}(\mathcal{R})\right)$ for $h$ small enough.

Lemma 4.20. For $h$ small enough, $W \subset \operatorname{ker}\left(\mathcal{S}_{h}\right) ;\left(\mathcal{S}_{h}\right)_{h}$ is uniformly bounded.

One can then prove an "orthogonality" result involving $\mathcal{S}_{h}$.

Proposition 4.21. For all $f$ in $L^{2}(\mathcal{R})$ and $\varphi_{h}$ in $W_{h}$, one has for $h$ small enough

$$
\left(\underline{\nu \Sigma_{f}} \mathcal{S}_{h} f, \varphi_{h}\right)_{0, \mathcal{R}}=0 .
$$

Proof. Let $f$ be in $L^{2}(\mathcal{R})$ and $\varphi_{h}$ be in $W_{h}$. We find:

$$
\begin{aligned}
\left(\underline{\nu \Sigma}_{f} \mathcal{S}_{h} f, \varphi_{h}\right)_{0, \mathcal{R}} & =\left(\underline{\nu \Sigma}_{f}\left(F_{h}^{-1} E_{h} f-f\right), \varphi_{h}\right)_{0, \mathcal{R}} \\
& =\left(\underline{\nu \Sigma}_{f}\left(F_{h}^{-1} E_{h} f-E_{h} f\right), \varphi_{h}\right)_{0, \mathcal{R}} \\
& =\left(\underline{\nu \Sigma}_{f}\left(F_{h}^{-1} E_{h} f-F_{h} F_{h}^{-1} E_{h} f\right), \varphi_{h}\right)_{0, \mathcal{R}}
\end{aligned}
$$

The second equality uses (4.38) with $\varphi=f$. One concludes by remarking that $\psi=F_{h}^{-1} E_{h} f \in W$ so $\left(\underline{\nu \Sigma_{f}}(\psi-\right.$ $\left.\left.F_{h} \psi\right), \varphi_{h}\right)_{0, \mathcal{R}}=0$ using again (4.38), because $F_{h} \psi=E_{h} \psi$.

To obtain an optimal rate of convergence we restrict the operators $B_{\mu}$ and $B_{\mu}^{h}$ to the eigenspace $W$. We denote finally by $\hat{B}_{\mu}$ and $\hat{B}_{\mu}^{h}$ the operators, from $W$ to itself, $\hat{B}_{\mu}=\left.B_{\mu}\right|_{W}$ and $\hat{B}_{\mu}^{h}=F_{h}^{-1} B_{\mu}^{h} F_{h}$. Let us estimate

$$
\left\|\hat{B}_{\mu}-\hat{B}_{\mu}^{h}\right\|_{\mathcal{L}(W)}=\sup _{\varphi, \varphi^{\prime} \in W \backslash\{0\}} \frac{\left|\left(\varphi,\left(\hat{B}_{\mu}-\hat{B}_{\mu}^{h}\right) \varphi^{\prime}\right)_{W}\right|}{\|\varphi\|_{W}\left\|\varphi^{\prime}\right\|_{W}} .
$$

Theorem 4.22. Let $\omega$ be as in Definition 4.15. Then for $h$ small enough, the following estimate holds true

$$
\left\|\hat{B}_{\mu}-\hat{B}_{\mu}^{h}\right\|_{\mathcal{L}(W)} \lesssim h^{2 \omega} .
$$

Proof. Using the definition of $F_{h}$, Lemma 4.18 and finally Lemma 4.20, one checks that for all $\varphi^{\prime} \in W$ :

$$
\begin{aligned}
\left(\hat{B}_{\mu}-\hat{B}_{\mu}^{h}\right) \varphi^{\prime} & =B_{\mu} \varphi^{\prime}-F_{h}^{-1} B_{\mu}^{h} F_{h} \varphi^{\prime} \\
& =B_{\mu} \varphi^{\prime}-F_{h}^{-1} B_{\mu}^{h} E_{h} \varphi^{\prime} \\
& =B_{\mu} \varphi^{\prime}-F_{h}^{-1} E_{h} B_{\mu}^{h} \varphi^{\prime} \\
& =\left(B_{\mu}-B_{\mu}^{h}\right) \varphi^{\prime}+B_{\mu}^{h} \varphi^{\prime}-F_{h}^{-1} E_{h} B_{\mu}^{h} \varphi^{\prime}+\mathcal{S}_{h} B_{\mu} \varphi^{\prime} \\
& =\left(B_{\mu}-B_{\mu}^{h}\right) \varphi^{\prime}+\mathcal{S}_{h}\left(B_{\mu}-B_{\mu}^{h}\right) \varphi^{\prime} .
\end{aligned}
$$

Hence, given $\varphi, \varphi^{\prime} \in W$, we can bound $\left|\left(\varphi,\left(\hat{B}_{\mu}-\hat{B}_{\mu}^{h}\right) \varphi^{\prime}\right)_{W}\right|=\left|\left(\underline{\nu \Sigma}_{f} \varphi,\left(\hat{B}_{\mu}-\hat{B}_{\mu}^{h}\right) \varphi^{\prime}\right)_{0, \mathcal{R}}\right|$ by

$$
\left|\left(\underline{\nu}_{f} \varphi,\left(B_{\mu}-B_{\mu}^{h}\right) \varphi^{\prime}\right)_{0, \mathcal{R}}\right|+\left|\left(\underline{\nu}_{f} \varphi, \mathcal{S}_{h}\left(B_{\mu}-B_{\mu}^{h}\right) \varphi^{\prime}\right)_{0, \mathcal{R}}\right|
$$

Let us bound each part separately below.

One obtains from the difference between (4.33) and (4.34)

$$
\begin{aligned}
\left({\left.\underline{\nu \Sigma_{f}} \varphi,\left(B_{\mu}-B_{\mu}^{h}\right) \varphi^{\prime}\right)_{0, \mathcal{R}}=} a\left(\left(A_{\mu}-A_{\mu}^{h}\right) \varphi,\left(A_{\mu}-A_{\mu}^{h}\right) \varphi^{\prime}\right)+b\left(\left(A_{\mu}-A_{\mu}^{h}\right) \varphi^{\prime},\left(B_{\mu}-B_{\mu}^{h}\right) \varphi\right)\right. \\
\\
+b\left(\left(A_{\mu}-A_{\mu}^{h}\right) \varphi,\left(B_{\mu}-B_{\mu}^{h}\right) \varphi^{\prime}\right)+t\left(\left(B_{\mu}-B_{\mu}^{h}\right) \varphi,\left(B_{\mu}-B_{\mu}^{h}\right) \varphi^{\prime}\right) .
\end{aligned}
$$


Then, one can bound the first part:

$$
\begin{aligned}
\left|\left(\underline{\nu \Sigma_{f}} \varphi,\left(B_{\mu}-B_{\mu}^{h}\right) \varphi^{\prime}\right)_{0, \mathcal{R}}\right| \lesssim & \left\|\left(A_{\mu}-A_{\mu}^{h}\right) \varphi\right\|_{0, \mathcal{R}}\left\|\left(A_{\mu}-A_{\mu}^{h}\right) \varphi^{\prime}\right\|_{0, \mathcal{R}} \\
& +\left\|\operatorname{div}\left(A_{\mu}-A_{\mu}^{h}\right) \varphi^{\prime}\right\|_{0, \mathcal{R}}\left\|\left(B_{\mu}-B_{\mu}^{h}\right) \varphi\right\|_{0, \mathcal{R}} \\
& +\left\|\operatorname{div}\left(A_{\mu}-A_{\mu}^{h}\right) \varphi\right\|_{0, \mathcal{R}}\left\|\left(B_{\mu}-B_{\mu}^{h}\right) \varphi^{\prime}\right\|_{0, \mathcal{R}} \\
& +\left\|\left(B_{\mu}-B_{\mu}^{h}\right) \varphi\right\|_{0, \mathcal{R}}\left\|\left(B_{\mu}-B_{\mu}^{h}\right) \varphi^{\prime}\right\|_{0, \mathcal{R}} \\
\lesssim & h^{2 \omega}\|\varphi\|_{W}\left\|\varphi^{\prime}\right\|_{W} .
\end{aligned}
$$

The second part is bounded by:

$$
\begin{aligned}
\left|\left(\underline{\nu \Sigma_{f}} \varphi, \mathcal{S}_{h}\left(B_{\mu}-B_{\mu}^{h}\right) \varphi^{\prime}\right)_{0, \mathcal{R}}\right| & =\left|\left(\underline{\nu \Sigma_{f}}\left(\varphi-F_{h} \varphi\right), \mathcal{S}_{h}\left(B_{\mu}-B_{\mu}^{h}\right) \varphi^{\prime}\right)\right| \\
& \leq\left\|\underline{\nu \Sigma_{f}}\left(\varphi-F_{h} \varphi\right)\right\|_{0, \mathcal{R}}\left\|\mathcal{S}_{h}\left(B_{\mu}-B_{\mu}^{h}\right) \varphi^{\prime}\right\|_{0, \mathcal{R}} \\
& \lesssim\left\|\underline{\nu \Sigma_{f}}\left(\varphi-F_{h} \varphi\right)\right\|_{0, \mathcal{R}}\left\|\left(B_{\mu}-B_{\mu}^{h}\right) \varphi^{\prime}\right\|_{0, \mathcal{R}} \\
& \lesssim h^{2 \omega}\|\varphi\|_{W}\left\|\varphi^{\prime}\right\|_{W} .
\end{aligned}
$$

In the first line we use Proposition 4.21 with $f=\left(B_{\mu}-B_{\mu}^{h}\right) \varphi^{\prime}$ and $\varphi_{h}=F_{h} \varphi$. In the third line we use the uniform continuity of $\mathcal{S}_{h}$ in $h$, and in the last line we use the first inequality of Proposition 4.17 and the estimation (4.39). Therefore we have obtained (4.40).

From this estimation and the work of Osborn in Theorem 2 of [27], one derives an optimal estimate on the error on the eigenvalues.

Corollary 4.23. Let $\omega$ be as in Definition 4.15. Then for $h$ small enough, the error on the eigenvalue is given by

$$
\left|\nu-\nu_{h}\right| \lesssim h^{2 \omega}
$$

Remark 4.24. If $\nu$ has an algebraic multiplicity $m_{\nu}>1$, the previous analysis and the a priori estimate are still valid with $\nu_{h}=\frac{1}{m_{\nu}} \sum_{i=1}^{m_{\nu}} \nu_{h, i}$, where $\left(\nu_{h, i}\right)_{i=1, m_{\nu}}$ are the $m$ discrete eigenvalues closest to $\nu$, see again Theorem 2 of [27].

\section{THE DD CASE}

We continue by considering the neutron diffusion problem using a domain decomposition method: we call it the DD case. The diffusion problem with low-regularity solution in a mixed, multi-domain form has been analyzed in [13]. In this section, we first define some notations and spaces. Then we recall some results of [13], in which technical aspects on the choice and properties of the spaces and discretization are discussed. Finally, we define the variational formulation. The numerical analysis of the DD case is carried out in Section 6 .

\subsection{Setting of the DD spaces}

Let us consider a partition $\left\{\widetilde{\mathcal{R}}_{i}\right\}_{1 \leq i \leq \widetilde{N}}$ of $\mathcal{R}$ which can be independent from the physical partition of the materials in $\mathcal{R}$ (see e.g. $[10,11,23]$ ). In other words, it can happen that $\left\{\widetilde{\mathcal{R}}_{i}\right\}_{1 \leq i \leq \tilde{N}} \neq\left\{\mathcal{R}_{i}\right\}_{1 \leq i \leq N}$. We denote by $\Gamma_{i j}$ the interface between two subdomains $\widetilde{\mathcal{R}}_{i}$ and $\widetilde{\mathcal{R}}_{j}$, for $i \neq j$ : if the Hausdorff dimension of $\overline{\widetilde{\mathcal{R}}_{i}} \cap \overline{\widetilde{\mathcal{R}}_{j}}$ is $d-1$, then $\Gamma_{i j}=\operatorname{int}\left(\widetilde{\mathcal{R}}_{i} \cap \overline{\widetilde{\mathcal{R}}_{j}}\right)$; otherwise, $\Gamma_{i j}=\emptyset$. By construction, $\Gamma_{i j}=\Gamma_{j i}$. We define the interface $\Gamma_{S}$, 
respectively the wirebasket $\partial \Gamma_{W}$ by

$$
\Gamma_{S}=\bigcup_{i=1}^{\widetilde{N}} \bigcup_{j=i+1}^{\widetilde{N}} \overline{\Gamma_{i j}}, \quad \partial \Gamma_{W}=\bigcup_{i=1}^{\widetilde{N}} \bigcup_{j=i+1}^{\widetilde{N}} \partial \Gamma_{i j}
$$

It is stressed that the resulting interface $\Gamma_{S}$ needs not necessarily coincide with the physical interface between cells.

When $d=2$, the wirebasket consists of isolated crosspoints. When $d=3$, the wirebasket consists of open edges and crosspoints. For a field $v$ defined over $\mathcal{R}$, we shall use the notation $v_{i}=v_{\mid \widetilde{\mathcal{R}}_{i}}$, for $1 \leq i \leq \widetilde{N}$. Let us define the function space with zero Dirichlet boundary condition:

$$
\widetilde{\mathcal{P}} H_{0}^{1}(\mathcal{R})=\left\{\psi \in L^{2}(\mathcal{R}) \mid \psi_{i} \in H^{1}\left(\widetilde{\mathcal{R}}_{i}\right), \psi_{\mid \partial \widetilde{\mathcal{R}}_{i} \backslash \overline{\Gamma_{S}}}=0,1 \leq i \leq \widetilde{N}\right\}
$$

When $\Gamma_{i j} \neq \emptyset$, let $H_{\Gamma_{i j}}^{1 / 2}$ be the set of $H^{1 / 2}\left(\Gamma_{i j}\right)$ functions whose continuation by 0 to $\partial \mathcal{R}_{i}$ belongs to $H^{1 / 2}\left(\partial \mathcal{R}_{i}\right)$. On can prove that $H_{\Gamma_{i j}}^{1 / 2}=H_{\Gamma_{j i}}^{1 / 2}$. We also introduce the space of piecewise $\mathbf{H}$ (div) vector-valued functions:

$$
\widetilde{\mathcal{P}} \mathbf{H}(\operatorname{div}, \mathcal{R})=\left\{\mathbf{q} \in \mathbf{L}^{2}(\mathcal{R}) \mid \mathbf{q}_{i} \in \mathbf{H}\left(\operatorname{div}, \widetilde{\mathcal{R}}_{i}\right), 1 \leq i \leq \widetilde{N}\right\}, \quad\|\mathbf{q}\|_{\widetilde{\mathcal{P}} \mathbf{H}(\operatorname{div}, \mathcal{R})}=\left(\sum_{i}\left\|\mathbf{q}_{i}\right\|_{\mathbf{H}\left(\operatorname{div}, \widetilde{\mathcal{R}}_{i}\right)}^{2}\right)^{1 / 2} .
$$

For $\mathbf{p} \in \widetilde{\mathcal{P}} \mathbf{H}(\operatorname{div}, \mathcal{R})$, let us set $[\mathbf{p} \cdot \mathbf{n}]_{i j}:=\sum_{k=i, j} \mathbf{p}_{k} \cdot \mathbf{n}_{k \mid \Gamma_{i j}}$ the jump of the normal component of $\mathbf{p}$ on $\Gamma_{i j}$ when $\Gamma_{i j} \neq \emptyset$. [p $\left.\cdot \mathbf{n}\right]_{i j}$ is well defined in $\left(H_{\Gamma_{i j}}^{1 / 2}\right)^{\prime}$ the dual space of $H_{\Gamma_{i j}}^{1 / 2}$ (see e.g. [19]). The global jump [p $\left.\cdot \mathbf{n}\right]$ of the normal component on the interface is defined by:

$$
[\mathbf{p} \cdot \mathbf{n}]_{\mid \Gamma_{i j}}:=[\mathbf{p} \cdot \mathbf{n}]_{i j}, \quad \text { for } 1 \leq i, j \leq \widetilde{N} .
$$

By definition, it holds $[\mathbf{p} \cdot \mathbf{n}] \in \prod_{i<j}\left(H_{\Gamma_{i j}}^{1 / 2}\right)^{\prime}$. We recall that for $\mathbf{p} \in \mathbf{H}(\operatorname{div}, \mathcal{R})$, the global jump vanishes: $[\mathbf{p} \cdot \mathbf{n}]=0$ (see e.g. [13], Lem. 1).

We introduce finally the following Hilbert spaces:

$$
\begin{aligned}
& M=\left\{\psi_{S} \in \prod_{i<j} L^{2}\left(\Gamma_{i j}\right)\right\},\left\|\psi_{S}\right\|_{M}=\left(\sum_{i<j}\left\|\psi_{S}\right\|_{0, \Gamma_{i j}}^{2}\right)^{1 / 2} ; \\
& H_{-}^{1 / 2}\left(\Gamma_{S}\right)=\left\{\psi_{S} \in M \mid \psi_{S \mid \Gamma_{i j}} \in H^{1 / 2}\left(\Gamma_{i j}\right), \forall i<j\right\}, \text { with graph norm; } \\
& \widetilde{\mathbf{Q}}=\{\mathbf{q} \in \widetilde{\mathcal{P}} \mathbf{H}(\operatorname{div}, \mathcal{R}) \mid[\mathbf{q} \cdot \mathbf{n}] \in M\}, \\
& \\
& \widetilde{q} \|_{\widetilde{\mathbf{Q}}}=\left(\|\mathbf{q}\|_{\widetilde{\mathcal{P}} \mathbf{H}(\operatorname{div}, \mathcal{R})}^{2}+\|[\mathbf{q} \cdot \mathbf{n}]\|_{M}^{2}\right)^{1 / 2} ; \\
& \widetilde{\mathbf{X}}=\left\{\xi:=(\mathbf{q}, \psi) \in \widetilde{\mathbf{Q}} \times L^{2}(\mathcal{R})\right\},\|\xi\|_{\widetilde{\mathbf{X}}}:=\left(\|\mathbf{q}\|_{\widetilde{\mathbf{Q}}}^{2}+\|\psi\|_{0, \mathcal{R}}^{2}\right)^{1 / 2} ; \\
& \mathrm{W}=\left\{\mathrm{w}:=\left(\xi, \psi_{S}\right) \in \widetilde{\mathbf{X}} \times M\right\},\|\mathrm{w}\|_{\mathrm{w}}:=\left(\|\xi\|_{\widetilde{\mathbf{X}}}^{2}+\left\|\psi_{S}\right\|_{M}^{2}\right)^{1 / 2} ;
\end{aligned}
$$

By construction, one has $M \subset \prod_{i<j}\left(H_{\Gamma_{i j}}^{1 / 2}\right)^{\prime}$. We will next define a variational formulation which is conforming in $\widetilde{\mathbf{Q}} \times L^{2}(\mathcal{R})$. 


\subsection{Variational formulation and discretization in the DD case}

The mixed form of the neutron diffusion problem (4.1) is now given by (see Sect. 3.2 from [13]):

Find $\left(\mathbf{p}, \phi, \phi_{S}\right) \in \widetilde{\mathbf{Q}} \times \widetilde{\mathcal{P}} H_{0}^{1}(\mathcal{R}) \times M$ such that:

$$
\begin{cases}-D_{i}^{-1} \mathbf{p}_{i}-\operatorname{grad} \phi_{i}=0 & \text { in } \widetilde{\mathcal{R}}_{i}, \quad \text { for } 1 \leq i \leq \widetilde{N} \\ \operatorname{div} \mathbf{p}_{i}+\Sigma_{a, i} \phi_{i}=S_{f, i} & \text { in } \widetilde{\mathcal{R}}_{i}, \quad \text { for } 1 \leq i \leq \widetilde{N} \\ \phi_{i}=\phi_{S} & \text { on } \partial \widetilde{\mathcal{R}}_{i} \cap \Gamma_{S}, \quad \text { for } 1 \leq i \leq \widetilde{N} \\ {[\mathbf{p} \cdot \mathbf{n}]=0} & \text { on } \Gamma_{S} .\end{cases}
$$

To solve this problem, we are looking for a solution $\left((\mathbf{p}, \phi), \phi_{S}\right)$ in W. Find $\left((\mathbf{p}, \phi), \phi_{S}\right) \in \mathrm{W}$, such that $\forall\left((\mathbf{q}, \psi), \psi_{S}\right) \in \mathrm{W}:$

$$
\int_{\mathcal{R}}\left(-D^{-1} \mathbf{p} \cdot \mathbf{q}+\phi \operatorname{div} \mathbf{q}+\psi \operatorname{div} \mathbf{p}+\Sigma_{a} \phi \psi\right)+\int_{\Gamma_{S}}[\mathbf{p} \cdot \mathbf{n}] \psi_{S}-\int_{\Gamma_{S}}[\mathbf{q} \cdot \mathbf{n}] \phi_{S}=\int_{\mathcal{R}} S_{f} \psi
$$

In (5.1)-(5.2), $\phi_{S}, \psi_{S}$ play the role of Lagrange multipliers, with $M$ the space of those Lagrange multipliers. To be mathematically precise, we should be integrating on $\cup_{i<j} \Gamma_{i j}$ instead of $\Gamma_{S}$. We make this slight abuse of notations from now on. This approach is called the $\mathrm{DD}+L^{2}$-jumps method.

From now on, we use the notations:

- $\mathrm{u}=\left(\zeta, \phi_{S}\right), \zeta=(\mathbf{p}, \phi), \mathbf{p}=\left(\mathbf{p}_{i}\right)_{1 \leq i \leq \widetilde{N}}$ and $\phi=\left(\phi_{i}\right)_{1 \leq i \leq \widetilde{N}}$

- $\mathbf{w}=\left(\xi, \psi_{S}\right), \xi=(\mathbf{q}, \psi), \mathbf{q}=\left(\mathbf{q}_{i}\right)_{1 \leq i \leq \tilde{N}}$ and $\psi=\left(\psi_{i}\right)_{1 \leq i \leq \tilde{N}} ;$

and we define the bilinear forms:

$$
\ell_{S}:\left\{\begin{array}{l}
\mathrm{W} \times \mathrm{W} \rightarrow \mathbb{R} \\
(\mathrm{u}, \mathrm{w}) \mapsto \int_{\Gamma_{S}}[\mathbf{p} \cdot \mathbf{n}] \psi_{S}
\end{array}\right.
$$

and:

$$
c_{S}:\left\{\begin{array}{l}
\mathrm{W} \times \mathrm{W} \rightarrow \mathbb{R} \\
(\mathrm{u}, \mathrm{w}) \mapsto c(\zeta, \xi)+\ell_{S}(\mathrm{u}, \mathrm{w})-\ell_{S}(\mathrm{w}, \mathrm{u})
\end{array} .\right.
$$

We consider the linear form:

$$
f_{S}:\left\{\begin{array}{l}
\mathrm{w} \rightarrow \mathbb{R} \\
\mathrm{w} \mapsto f(\xi)
\end{array}\right.
$$

Above, we extended the definition (4.7) (resp. (4.8)) of the form $c$ (resp. f), to elements of $\widetilde{\mathbf{X}} \times \widetilde{\mathbf{X}}$ (resp. $\widetilde{\mathbf{X}}$ ). We may rewrite the variational formulation (5.2) as:

Find $\mathrm{u} \in \mathrm{W}$ such that $\forall \mathrm{w} \in \mathrm{W}$ :

$$
c_{S}(\mathrm{u}, \mathrm{w})=f_{S}(\mathrm{w}) .
$$

We recall that $c_{S}$ satisfies an inf-sup condition, so the variational problem is well-posed (see [13], Sect. 4), and that, under the assumptions of Proposition 3.1, the global jump of $\mathbf{p}$ vanishes: $[\mathbf{p} \cdot \mathbf{n}]=0$ in $M$ (see [13], Lem. 1).

We study abstract, conforming, discretization of the variational formulation (5.6) as it is done in Section 5 from 
[13]. To that aim, we introduce discrete, finite-dimensional, spaces indexed by a (small) parameter $h$ as follows: $\mathbf{Q}_{i, h} \subset \mathbf{H}\left(\operatorname{div}, \widetilde{\mathcal{R}}_{i}\right)$ and $L_{i, h} \subset L^{2}\left(\widetilde{\mathcal{R}}_{i}\right)$, for $1 \leq i \leq \widetilde{N}$. We impose the following requirements, for all $1 \leq i \leq \widetilde{N}$ :

- $\mathbf{q}_{i, h} \cdot \mathbf{n}_{\mid \partial \widetilde{\mathcal{R}}_{i}} \in L^{2}\left(\partial \widetilde{\mathcal{R}}_{i}\right)$ for all $h>0$, for all $\mathbf{q}_{i, h} \in \mathbf{Q}_{i, h}$;

- $\operatorname{div} \mathbf{Q}_{i, h} \subset L_{i, h}$ for all $h>0$;

- $\left(\mathbf{Q}_{i, h}\right)_{h}$ and $\left(L_{i, h}\right)_{h}$ satisfy the approximability property $(4.12)$ in $\widetilde{\mathcal{R}}_{i}$.

Then, let

$$
\widetilde{\mathbf{Q}}_{h}=\prod_{1 \leq i \leq \widetilde{N}} \mathbf{Q}_{i, h} \quad \text { and } \quad L_{h}=\prod_{1 \leq i \leq \widetilde{N}} L_{i, h}
$$

In particular, the discretization $\widetilde{\mathbf{Q}}_{h} \times L_{h}$ is globally conforming in $\widetilde{\mathbf{Q}} \times L^{2}(\mathcal{R})$. We endow $\widetilde{\mathbf{Q}}_{h}$ with the norm $\|\cdot\|_{\widetilde{\mathbf{Q}}}$, while $L_{h}$ is endowed with $\|\cdot\|_{0, \mathcal{R}}$.

We then define $T_{i, h}$ as the space of the normal traces of vectors of $\mathbf{Q}_{i, h}$ on $\partial \widetilde{\mathcal{R}}_{i} \cap \Gamma_{S}$ :

$$
T_{i, h}:=\left\{q_{i, h} \in L^{2}\left(\partial \widetilde{\mathcal{R}}_{i} \cap \Gamma_{S}\right) \mid \exists \mathbf{q}_{i, h} \in \mathbf{Q}_{i, h}, q_{i, h}=\mathbf{q}_{i, h} \cdot \mathbf{n}_{i \mid \partial \widetilde{\mathcal{R}}_{i} \cap \Gamma_{S}}\right\}
$$

Classically, several situations can occur on a given interface $\Gamma_{i j}, 1 \leq i, j \leq \tilde{N}$ :

(1) non-nested meshes: $T_{i, h \mid \Gamma_{i j}} \not \subset T_{j, h \mid \Gamma_{i j}}$ and $T_{j, h \mid \Gamma_{i j}} \not \subset T_{i, h \mid \Gamma_{i j}}$;

(2) nested meshes: $T_{i, h \mid \Gamma_{i j}} \subset T_{j, h \mid \Gamma_{i j}}$ or $T_{j, h \mid \Gamma_{i j}} \subset T_{i, h \mid \Gamma_{i j}}$;

(3) matching meshes: nested meshes with $T_{i, h \mid \Gamma_{i j}}=T_{j, h \mid \Gamma_{i j}}$.

Usually, the term nested meshes is used to describe a family of successively refined meshes. In this paper, we will use this expression to express that on all interfaces $\Gamma_{i j}$, case (5.2) described above holds. As an illustration, see the interfaces between the subdomains in Fig. 3a.

Let us denote by $M_{h} \subset M$ the discrete space of the Lagrange multipliers. We assume that $M_{h}$ includes the subspace $M_{h}^{0}$ of piecewise constant fields. We introduce the discrete projection operators ([13], Sect. 5) from the spaces of normal traces $T_{i, h}$ to $M_{h}$, and vice versa, which are defined by:

$$
\forall q_{i, h} \in T_{i, h}, \forall \psi_{S, h} \in M_{h}\left\{\begin{array}{l}
\int_{\partial \widetilde{\mathcal{R}}_{i} \cap \Gamma_{S}}\left(\Pi_{i}\left(q_{i, h}\right)-q_{i, h}\right) \psi_{S, h}=0 \\
\int_{\partial \widetilde{\mathcal{R}}_{i} \cap \Gamma_{S}}\left(\pi_{i}\left(\psi_{S, h}\right)-\psi_{S, h}\right) q_{i, h}=0
\end{array} .\right.
$$

As the operators $\Pi_{i}$ and $\pi_{i}$ are orthogonal projections, they are continuous, with a continuity modulus equal to 1 . We also introduce the orthogonal projection operator $\Pi_{S}^{0}: M \rightarrow M_{h}^{0}$. According to Proposition 1.135 of [17], if we denote by $h_{S}$ the meshsize on $\Gamma_{S}$ :

$$
\forall \psi_{S} \in H_{-}^{1 / 2}\left(\Gamma_{S}\right),\left\|\psi_{S}-\Pi_{S}^{0}\left(\psi_{S}\right)\right\|_{M} \lesssim h_{S}^{1 / 2}\left\|\psi_{S}\right\|_{H_{-}^{1 / 2}\left(\Gamma_{S}\right)}
$$

Next, let $\mathbf{p}_{h} \in \widetilde{\mathbf{Q}}_{h}$. We define the discrete jump of the normal component of $\mathbf{p}_{h}$ on the interface $\Gamma_{i j}$ as $\left[\mathbf{p}_{h}\right.$. $\mathbf{n}]_{h, i j}:=\sum_{l=i, j} \Pi_{l}\left(\mathbf{p}_{l, h} \cdot \mathbf{n}_{l \mid \Gamma_{i j}}\right)$. The discrete global jump of the normal component, $\left[\mathbf{p}_{h} \cdot \mathbf{n}\right]_{h} \in M_{h}$, is defined by:

$$
\left[\mathbf{p}_{h} \cdot \mathbf{n}\right]_{h \mid \Gamma_{i j}}:=\left[\mathbf{p}_{h} \cdot \mathbf{n}\right]_{h, i j}, \text { for } 1 \leq i, j \leq \tilde{N}
$$


We finally define:

$$
\begin{aligned}
\widetilde{\mathbf{X}}_{h} & =\left\{\xi_{h}:=\left(\mathbf{q}_{h}, \psi_{h}\right) \in \widetilde{\mathbf{Q}}_{h} \times L_{h}\right\}, \text { endowed with }\|\cdot\|_{\tilde{\mathbf{X}}}, \\
\mathrm{W}_{h} & =\left\{\mathrm{w}_{h}:=\left(\xi_{h}, \psi_{S, h}\right) \in \widetilde{\mathbf{X}}_{h} \times M_{h}\right\}, \text { endowed with }\|\cdot\|_{\mathrm{w}} .
\end{aligned}
$$

In the $\mathrm{DD}+L^{2}$-jumps setting, the conforming discretization of the variational formulation (5.6) reads:

Find $\mathrm{u}_{h} \in \mathrm{w}_{h}$ such that $\forall \mathrm{w}_{h} \in \mathrm{w}_{h}, c_{S}\left(\mathrm{u}_{h}, \mathrm{w}_{h}\right)=f_{S}\left(\mathrm{w}_{h}\right)$.

It is shown in Section 5 from [13] that $c_{S}$ verifies a discrete inf-sup condition if the following conditions hold:

$$
\exists \beta_{h}>0, \forall \mathbf{q}_{h} \in \widetilde{\mathbf{Q}}_{h}, \int_{\Gamma_{S}}\left[\mathbf{q}_{h} \cdot \mathbf{n}\right]_{h}\left[\mathbf{q}_{h} \cdot \mathbf{n}\right] \geq \beta_{h} \int_{\Gamma_{S}}\left[\mathbf{q}_{h} \cdot \mathbf{n}\right]^{2}
$$

and

$$
\begin{aligned}
& \exists \gamma_{h}>0, \quad \forall \psi_{S, h} \in M_{h}, \\
& \sum_{i=1}^{\widetilde{N}} \sum_{j=i+1}^{\widetilde{N}} \int_{\Gamma_{i j}}\left(\pi_{i}\left(\psi_{S, h}\right)^{2}+\pi_{j}\left(\psi_{S, h}\right)^{2}\right) \geq \gamma_{h}\left\|\psi_{S, h}\right\|_{M}^{2},
\end{aligned}
$$

Moreover, if $\beta_{h}$ and $\gamma_{h}$ can be chosen independently of $h$, the form $c_{S}$ satisfies a udisc. For instance, conditions (5.11)-(5.12) are uniformly fulfilled when $M_{h}$ is chosen as

$$
M_{h}=\sum_{i=1}^{\widetilde{N}} T_{i, h} .
$$

Last, under (5.11), one easily checks that $\left[\mathbf{p}_{h} \cdot \mathbf{n}\right]=0$. In other words:

$$
\mathbf{p}_{h} \in \mathbf{H}(\operatorname{div}, \mathcal{R}) \cap \widetilde{\mathbf{Q}}_{h} .
$$

In the DD case, we define $\mathbf{Q}_{h}=\mathbf{H}(\operatorname{div}, \mathcal{R}) \cap \widetilde{\mathbf{Q}}_{h}$.

\section{Numerical ANALYSIS IN THE DD CASE}

To carry out the numerical analysis in the low-regularity case, we first introduce a suitable discretization of the DD problem, and then we carry out the numerical analysis on this discretization. Again, if one chooses another discretization that fulfills those properties detailed in the previous section, one may recover similar convergence results.

\subsection{Discretization}

We consider (5.10) where the RTN finite element is used on each subdomain with a conforming mesh, or triangulation. For $1 \leq i \leq \widetilde{N}$, let $h_{i}$ denote the local meshsize in $\widetilde{\mathcal{R}}_{i}$, and $h=\max _{i} h_{i}$ the global meshsize. Let us denote by $k_{i} \geq 0$ the order of the discretization in $\widetilde{\mathcal{R}}_{i}$, and $k=\min _{i} k_{i}$, the minimal order of the RTN finite element. The local RTN finite element subspace of $\mathbf{H}\left(\operatorname{div}, \widetilde{\mathcal{R}}_{i}\right) \times L^{2}\left(\widetilde{\mathcal{R}}_{i}\right)$ is defined as $\mathbf{Q}_{i, h_{i}}^{k_{i}} \times L_{i, h_{i}}^{k_{i}}$. With this choice, we have $\operatorname{div} \mathbf{Q}_{i, h_{i}}^{k_{i}} \subset L_{i, h_{i}}^{k_{i}}$ as required: local consistency is ensured. Now, if we set $\widetilde{\mathbf{Q}}_{h}^{k}=\prod_{1 \leq i \leq \widetilde{N}} \mathbf{Q}_{i, h_{i}}^{k_{i}}$ and $L_{h}^{k}=\prod_{1 \leq i \leq \widetilde{N}} L_{i, h_{i}}^{k_{i}}$, we have $\mathbf{q}_{i, h} \cdot \mathbf{n}_{\mid \partial \widetilde{\mathcal{R}}_{i}} \in L^{2}\left(\partial \widetilde{\mathcal{R}}_{i}\right)$ for all $\mathbf{q}_{i, h} \in \mathbf{Q}_{i, h_{i}}^{k_{i}}$, hence it follows that $\widetilde{\mathbf{Q}}_{h}^{k} \subset \widetilde{\mathbf{Q}}$ : 
the discretization $\widetilde{\mathbf{Q}}_{h}^{k} \times L_{h}^{k}$ is globally conforming in $\widetilde{\mathbf{Q}} \times L^{2}(\mathcal{R})$. For the reader's convenience, we omit the superscript $k_{i}$ in the analysis below.

Finally, we choose $M_{h}$ so that on the one hand (5.11)-(5.12) hold uniformly, and on the other hand it holds $h_{S} \lesssim h$ : we refer to Section 5.2 from [13] for an extended discussion on suitable choices. According to the first Strang's Lemma [17] and because $c_{S}$ verifies a udisc, the error reads:

$$
\left\|\mathrm{u}-\mathrm{u}_{h}\right\|_{\mathrm{W}} \lesssim \inf _{\mathrm{w}_{h} \in \mathrm{W}_{h}}\left\|\mathrm{u}-\mathrm{w}_{h}\right\|_{\mathrm{W}}
$$

As a consequence $\lim _{h \rightarrow 0}\left\|\mathrm{u}-\mathrm{u}_{h}\right\|_{\mathrm{w}}=0$. This result holds for nested and non-nested meshes. We study below how to improve the bound on the error, how to derive an Aubin-Nitsche estimate, and finally how to prove convergence for the generalized eigenvalue problem, for nested meshes. ${ }^{3}$ As previously, those results hold under

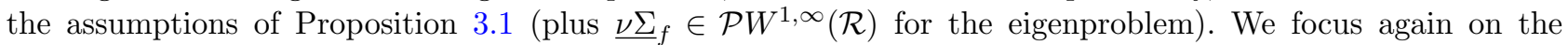
low-regularity case.

\subsection{A priori error estimates}

Let $\mathbf{q} \in \mathbf{H}(\operatorname{div}, \mathcal{R}) \cap \widetilde{\mathcal{P}} \mathbf{H}^{\mu}(\mathcal{R})$, with $0<\mu$. A global RTN interpolant of $\mathbf{q}$ is defined on every subdomain $\widetilde{\mathcal{R}}_{i}$ via its restriction $\mathbf{q}_{i}$, and denoted by $\tilde{\mathbf{q}}_{i, R}$ for $1 \leq i \leq \widetilde{N}$. One may thus define the global interpolant of $\mathbf{q}$ in $\widetilde{\mathbf{Q}}_{h}$, denoted by $\tilde{\mathbf{q}}_{R}$ henceforth: $\tilde{\mathbf{q}}_{R \mid \widetilde{\mathcal{R}}_{i}}=\tilde{\mathbf{q}}_{i, R}$ for $1 \leq i \leq \widetilde{N}$. Below, we also use the orthogonal projection operators $\pi^{0}: L^{2}(\mathcal{R}) \rightarrow L_{h}^{0}$ (see Sect. 4.5.1) and $\Pi_{S}^{0}: M \rightarrow M_{h}^{0}$ (see Sect. 5.2). One has the following result, whose proof is given in Appendix A.

Lemma 6.1. Assume that the meshes are nested, non-matching, on the interface $\Gamma_{f c}$, and that they are quasiuniform on $\Gamma_{f c}$. To fix ideas, we assume $T_{c, h \mid \Gamma_{f c}} \subset T_{f, h \mid \Gamma_{f c}}$ with $\left.T_{c, h \mid \Gamma_{f c}} \neq T_{f, h \mid \Gamma_{f c}}{ }^{4}\right)$.

Let $\mathbf{q} \in \mathbf{H}(\operatorname{div}, \mathcal{R}) \cap \mathbf{H}^{\mu}(\mathcal{R})$ with $0<\mu<1 / 2$, it holds:

$$
\left\|\left[\tilde{\mathbf{q}}_{R} \cdot \mathbf{n}\right]\right\|_{0, \Gamma_{f c}} \lesssim h_{f}^{1 / 2}\left\|\mathbf{q}_{f}\right\|_{\mathbf{H}\left(\operatorname{div}, \widetilde{\mathcal{R}}_{f}\right)} .
$$

Theorem 6.2. Let the assumptions of Proposition 3.1 hold, with $r_{\max }<1 / 2$. One has for matching meshes:

$$
\begin{aligned}
& \forall \mu \in] 0, r_{\max }\left[, \quad \forall S_{f} \in H^{\mu}(\mathcal{R}),\right. \\
& \left\|\mathbf{p}-\mathbf{p}_{h}\right\|_{\mathbf{H}(\operatorname{div}, \mathcal{R})}+\left\|\phi-\phi_{h}\right\|_{0, \mathcal{R}}+\left\|\phi_{S}-\phi_{S, h}\right\|_{M} \lesssim h^{\mu}\left\|S_{f}\right\|_{\mu, \mathcal{R}} .
\end{aligned}
$$

For nested, non-matching meshes, the result holds under the assumption that on an interface $\Gamma_{i j}$ where the meshes $T_{i, h \mid \Gamma_{i j}}$ and $T_{j, h \mid \Gamma_{i j}}$ are non-matching $\left(T_{i, h \mid \Gamma_{i j}} \neq T_{j, h \mid \Gamma_{i j}}\right)$, the families of triangulations of $T_{i, h \mid \Gamma_{i j}}$ and $T_{j, h \mid \Gamma_{i j}}$ are quasi-uniform.

Proof. We bound the different contributions in the right-hand side of (6.1) for some appropriately chosen discrete field $\mathrm{w}_{h}$. Recall that $\mathrm{u}=\left((\mathbf{p}, \phi), \phi_{S}\right)$.

Matching meshes. We know that $[\mathbf{p} \cdot \mathbf{n}]=0$. For matching meshes, one has also $\left[\tilde{\mathbf{p}}_{R} \cdot \mathbf{n}\right]=0$, so $\left[\left(\mathbf{p}-\tilde{\mathbf{p}}_{R}\right) \cdot \mathbf{n}\right]=0$. Starting from (6.1), the conclusion follows. Indeed, according to the a priori estimates (4.15), (4.20) and (5.9), $\mathrm{w}_{h}=\left(\tilde{\mathbf{p}}_{R}, \pi^{0} \phi, \Pi_{S}^{0}\left(\phi_{S}\right)\right) \in \mathrm{W}_{h}$ is such that

$$
\begin{aligned}
\left\|\mathrm{u}-\mathrm{w}_{h}\right\|_{\mathrm{W}}^{2} & =\sum_{i=1}^{\widetilde{N}}\left\|\mathbf{p}_{i}-\mathbf{p}_{i, R}\right\|_{\mathbf{H}\left(\operatorname{div}, \widetilde{\mathcal{R}}_{i}\right)}^{2}+\left\|\phi-\pi^{0} \phi\right\|_{0, \mathcal{R}}^{2}+\left\|\phi_{S}-\Pi_{S}^{0}\left(\phi_{S}\right)\right\|_{M}^{2} \\
& \lesssim h^{2 \mu}\left(|\mathbf{p}|_{\mu, \mathcal{R}}^{2}+\|\operatorname{div} \mathbf{p}\|_{\mu, \mathcal{R}}^{2}\right)+h^{2}\|\phi\|_{\mathcal{P} H^{1}(\mathcal{R})}^{2}+h_{S}\left\|\phi_{S}\right\|_{H_{-}^{1 / 2}\left(\Gamma_{S}\right)}^{2} \lesssim h^{2 \mu}\left\|S_{f}\right\|_{\mu, \mathcal{R}}^{2} .
\end{aligned}
$$

\footnotetext{
${ }^{3}$ For non-nested meshes, numerical illustrations suggest that the convergence properties can be recovered in some situations (see [13], Tab. 2). See also Section 6.5.

${ }^{4} f$ refers to fine discretization, while $c$ refers to coarse discretization.
} 
Hence we conclude that for matching meshes it holds:

$$
\left\|\mathrm{u}-\mathrm{u}_{h}\right\|_{\mathrm{W}} \lesssim h^{\mu}\left\|S_{f}\right\|_{\mu, \mathcal{R}}
$$

Nested meshes. In this case, $\left[\tilde{\mathbf{p}}_{R} \cdot \mathbf{n}\right] \neq 0$ in general. Nonetheless, one can use the result of Lemma 6.1 , to find that

$$
\left\|\left[\left(\mathbf{p}-\tilde{\mathbf{p}}_{R}\right) \cdot \mathbf{n}\right]\right\|_{M} \lesssim h^{1 / 2}\|\mathbf{p}\|_{\mathbf{H}(\operatorname{div}, \mathcal{R})},
$$

provided that the meshes are quasi-uniform on the part of the interface where they are non-matching. One concludes that the estimate (6.3) still holds for nested meshes under this condition.

Conclusion. Noting that it always holds $[\mathbf{p} \cdot \mathbf{n}]=\left[\mathbf{p}_{h} \cdot \mathbf{n}\right]=0$ (cf. (5.14)), developing the norm $\left\|\mathbf{u}-\mathrm{u}_{h}\right\|_{\mathrm{W}}$, one concludes:

$$
\left\|\mathbf{p}-\mathbf{p}_{h}\right\|_{\mathbf{H}(\operatorname{div}, \mathcal{R})}+\left\|\phi-\phi_{h}\right\|_{0, \mathcal{R}}+\left\|\phi_{S}-\phi_{S, h}\right\|_{M} \lesssim h^{\mu}\left\|S_{f}\right\|_{\mu, \mathcal{R}}
$$

In other words, we have the a priori error estimate (6.2).

As in the plain case, for "smooth data" $S_{f}$, i.e. $S_{f} \in H^{r_{\max }}(\mathcal{R})$, one expects a convergence rate at least in $h^{r_{\max }}$.

Remark 6.3. Within our framework, we obtain error estimates that generalize those of $[11,32]$ for low-regularity solutions. In addition, the technical aspects we propose remain quite simple and natural.

\subsection{Aubin-Nitsche-type estimates}

To derive improved estimates on the error $\left\|\phi-\phi_{h}\right\|_{0, \mathcal{R}}$, we adapt the calculations of Section 4.5.2 to the DD case. Recall that $\mathbf{Q}_{h}=\widetilde{\mathbf{Q}}_{h} \cap \mathbf{H}(\operatorname{div}, \mathcal{R})$. We already know that when conditions (5.11)-(5.12) hold, the solution $\left(\left(\mathbf{p}_{h}, \phi_{h}\right), \phi_{S, h}\right) \in \widetilde{\mathbf{X}}_{h} \times M_{h}$ of (5.10) (discrete DD case) is such that $\left(\mathbf{p}_{h}, \phi_{h}\right) \in \mathbf{X}_{h}$, since $\mathbf{p}_{h} \in \mathbf{Q}_{h}$. Then restricting the test-fields in (5.10) to elements of $\mathbf{X}_{h} \times M_{h}$ we observe that $\left(\mathbf{p}_{h}, \phi_{h}\right)$ satisfies (4.14) too (discrete plain-case), because all interface terms vanish. Hence, to estimate $\left\|\phi-\phi_{h}\right\|_{0, \mathcal{R}}$ in the DD case, we explicitly consider that the discrete fields $\left(\mathbf{p}_{h}, \phi_{h}\right)$ are also the solution to the variational formulation of the plain-case (4.14). Let us begin by a technical result, whose proof is given in Appendix A.

Lemma 6.4. Let the assumptions of Lemma 6.1 hold. Let $\mathbf{q} \in \mathbf{H}(\operatorname{div}, \mathcal{R}) \cap \mathbf{H}^{\mu}(\mathcal{R})$ with $0<\mu<1 / 2$, and define $\delta \mathbf{q}_{f c} \in \mathbf{Q}_{f, h}$ by $\delta \mathbf{q}_{f c} \cdot \mathbf{n}_{\mid \Gamma_{f c}}=\left(\tilde{\mathbf{q}}_{c, R} \cdot \mathbf{n}-\tilde{\mathbf{q}}_{f, R} \cdot \mathbf{n}\right)_{\mid \Gamma_{f c}}$ and zero extension in $\widetilde{\mathcal{\mathcal { R }}_{f}} \backslash \Gamma_{f c}$. It holds

$$
\left\|\delta \mathbf{q}_{f c}\right\|_{\mathbf{H}\left(\operatorname{div}, \widetilde{\mathcal{R}}_{f}\right)} \lesssim h^{\mu}\left(\left\|\mathbf{q}_{f}\right\|_{\mu, \widetilde{\mathcal{R}}_{f}}+\left\|\operatorname{div} \mathbf{q}_{f}\right\|_{0, \widetilde{\mathcal{R}}_{f}}\right) .
$$

Theorem 6.5. Under the assumptions of Theorem 6.2 with $r_{\max }<1 / 2$, one has for nested meshes:

$$
\forall \mu \in] 0, r_{\max }\left[, \quad \forall S_{f} \in H^{\mu}(\mathcal{R}), \quad\left\|\phi-\phi_{h}\right\|_{0, \mathcal{R}} \lesssim h^{2 \mu}\left\|S_{f}\right\|_{\mu, \mathcal{R}}\right.
$$

Proof. Matching meshes. In this case, one can use the theory already developed in Section 4.5 for the plain case, to conclude that (6.4) holds.

Nested meshes. The difficulty for non-matching meshes is that one cannot define the global RTN-interpolant of $\mathbf{p}$ directly. Instead it is defined via its subdomain interpolants $\left(\widetilde{\mathbf{p}}_{i, R}\right)_{1 \leq i \leq \widetilde{N}}$. Introduce, for $1 \leq i \leq \widetilde{N}, \mathcal{I}_{i}$ as the set of indices $j$ such that $T_{j, h \mid \Gamma_{i j}} \subset T_{i, h \mid \Gamma_{i j}}$ (since we are dealing with nested meshes, it holds $T_{j, h \mid \Gamma_{i j}} \subset T_{i, h \mid \Gamma_{i j}}$ or $\left.T_{i, h \mid \Gamma_{i j}} \subset T_{j, h \mid \Gamma_{i j}}\right)$. We proceed as follows to obtain an $\mathbf{H}(\operatorname{div}, \mathcal{R})$-conforming approximant, i.e. an element of $\mathbf{Q}_{h}$. On all interfaces $\Gamma_{i j}$, introduce $\delta \mathbf{p}_{i j} \cdot \mathbf{n}=\tilde{\mathbf{p}}_{c, R} \cdot \mathbf{n}_{\mid \Gamma_{i j}}-\tilde{\mathbf{p}}_{f, R} \cdot \mathbf{n}_{\mid \Gamma_{i j}}$ where $\tilde{\mathbf{p}}_{f, R}$ is the interpolant from the finer discretization on $\Gamma_{i j}$, resp. $\tilde{\mathbf{p}}_{c, R}$ is the interpolant from the coarser discretization on $\Gamma_{i j}$. By construction, 
$\delta \mathbf{p}_{i j} \cdot \mathbf{n}=0$ when $T_{i, h \mid \Gamma_{i j}}=T_{j, h \mid \Gamma_{i j}}$. Then $\delta \mathbf{p}_{i j} \cdot \mathbf{n}$ is extended by zero in $\widetilde{\mathcal{R}}_{i}$ to define an element of $\mathbf{Q}_{i, h}$; with a slight abuse of notation, we still denote the extension by $\delta \mathbf{p}_{i j}$. The $\mathbf{H}(\operatorname{div}, \mathcal{R})$-conforming approximant $\mathbf{p}_{R} \in \mathbf{Q}_{h}$ is then defined subdomain by subdomain as

$$
\mathbf{p}_{i, R}=\widetilde{\mathbf{p}}_{i, R}+\sum_{j \in \mathcal{I}_{i}} \delta \mathbf{p}_{i j} \quad \text { for } 1 \leq i \leq \tilde{N}
$$

Indeed, $\left[\mathbf{p}_{R} \cdot \mathbf{n}\right]_{\Gamma_{i j}}=0$ for $1 \leq i, j \leq \widetilde{N}$ by direct inspection. It remains to evaluate

$$
\begin{aligned}
& \left\|\mathbf{p}-\mathbf{p}_{R}\right\|_{\mathbf{H}(\operatorname{div}, \mathcal{R})}^{2}=\sum_{1 \leq i \leq \widetilde{N}}\left\|\mathbf{p}_{i}-\mathbf{p}_{i, R}\right\|_{\mathbf{H}\left(\operatorname{div}, \widetilde{\mathcal{R}}_{i}\right)}^{2}, \text { with } \\
& \left\|\mathbf{p}_{i}-\mathbf{p}_{i, R}\right\|_{\mathbf{H}\left(\operatorname{div}, \widetilde{\mathcal{R}}_{i}\right)} \leq\left\|\mathbf{p}_{i}-\widetilde{\mathbf{p}}_{i, R}\right\|_{\mathbf{H}\left(\operatorname{div}, \widetilde{\mathcal{R}}_{i}\right)}+\sum_{j \in \mathcal{I}_{i}}\left\|\delta \mathbf{p}_{i j}\right\|_{\mathbf{H}\left(\operatorname{div}, \widetilde{\mathcal{R}}_{i}\right)} \quad \text { for } 1 \leq i \leq \widetilde{N} .
\end{aligned}
$$

Above, the fact that the index $j$ belongs to $\mathcal{I}_{i}$ implies that if $\delta \mathbf{p}_{i j} \neq 0$, then the finer discretization on $\Gamma_{i j}$ automatically originates from $\widetilde{\mathcal{R}}_{i}$. To evaluate $\left\|\delta \mathbf{p}_{i j}\right\|_{\mathbf{H}\left(\operatorname{div}, \widetilde{\mathcal{R}}_{i}\right)}$, one uses the results of Lemma 6.4 to find

$$
\left\|\delta \mathbf{p}_{i j}\right\|_{\mathbf{H}\left(\operatorname{div}, \widetilde{\mathcal{R}}_{i}\right)} \lesssim h^{\mu}\left(\left\|\mathbf{p}_{i}\right\|_{\mu, \widetilde{\mathcal{R}}_{i}}+\left\|\operatorname{div} \mathbf{p}_{i}\right\|_{0, \widetilde{\mathcal{R}}_{i}}\right)
$$

Again, this bound holds under the condition that the meshes are quasi-uniform on the part of the interface where they are non-matching. Due to (4.20), one has $\left\|\mathbf{p}_{i}-\mathbf{p}_{i, R}\right\|_{\mathbf{H}\left(\operatorname{div}, \widetilde{\mathcal{R}}_{i}\right)} \lesssim h^{\mu}\left\|S_{f}\right\|_{\mu, \mathcal{R}}$ for $1 \leq i \leq \widetilde{N}$, and it follows that

$$
\left\|\mathbf{p}-\mathbf{p}_{R}\right\|_{\mathbf{H}(\operatorname{div}, \mathcal{R})} \lesssim h^{\mu}\left\|S_{f}\right\|_{\mu, \mathcal{R}}
$$

As a consequence (follow Sect. 4.5.2) we conclude that the estimate (6.4) holds.

\subsection{Numerical analysis of the generalized eigenvalue problem}

Let us focus on the approximation of the generalized eigenvalue problem (3.2) for low-regularity solutions with nested (matching or non-matching) meshes. We will follow the methodology of Section 4.6.

\subsubsection{Convergence in operator norm}

Let $0 \leq \mu<r_{\max }$ be given, we introduce an operator $B_{\mu}$ associated to the source problem (5.6): given $f \in$ $H^{\mu}(\mathcal{R})$, we call $B_{\mu} f=\phi \in H^{1}(\mathcal{R})$ the second component of the triple $\left(\mathbf{p}, \phi, \phi_{S}\right)$ that solves the source problem with $S_{f}=\underline{\nu \Sigma_{f} f}$. For the same reason as in the plain case Section 4.6.1, $B_{\mu}$ is a bounded and compact operator. Next, let us consider the discrete operator $B_{\mu}^{h}$ associated to the discrete source problem: given $f \in H^{\mu}(\mathcal{R})$, we

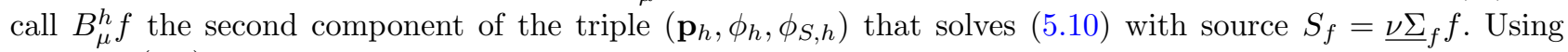
estimate (6.4), we obtain, like in the plain case, the result below.

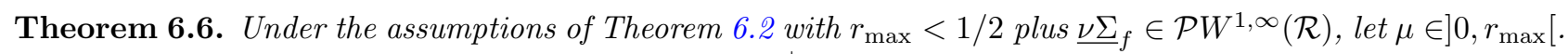
Provided that the families of triangulations are regular ${ }^{+}$on every subdomain, one has for nested meshes:

$$
\left\|B_{\mu}-B_{\mu}^{h}\right\|_{\mathcal{L}\left(H^{\mu}(\mathcal{R})\right)} \lesssim h^{\widetilde{\theta} \mu}
$$

where $\widetilde{\theta}=\min _{i=1}^{\widetilde{N}} \theta_{i}>0$, and for $1 \leq i \leq \widetilde{N}, \theta_{i}$ is defined by (4.29) on $\widetilde{\mathcal{R}}_{i}$.

We conclude to the absence of spectral pollution. 


\subsubsection{Optimal convergence rate}

Let the assumptions of Theorems 6.2 and 6.6 hold, and in particular the conditions for nested, non-matching meshes. We use the same notations as in Section 4.6.2. In particular, let $\widetilde{\omega}_{\nu}>0$ be the regularity exponent associated to $\nu$ with respect to $\left(\widetilde{\mathcal{P}} H^{1+s}(\mathcal{R})\right)_{s>0}$, and introduce $\widetilde{\omega}=\min \left(\widetilde{\omega}_{\nu}, k+1\right)$.

Let $\mu \in\left[0, r_{\max }\left[\right.\right.$ be given. As we defined $B_{\mu}\left(\right.$ resp. $\left.B_{\mu}^{h}\right)$, we define $A_{\mu}$ and $C_{\mu}$ (resp. $A_{\mu}^{h}$ and $\left.C_{\mu}^{h}\right)$ : for $f \in H^{\mu}(\mathcal{R})$, we call $A_{\mu} f=\mathbf{p} \in \widetilde{\mathbf{Q}}$ and $C_{\mu} f=\phi_{S} \in M$ (resp. $A_{\mu}^{h} f=\mathbf{p}_{h} \in \widetilde{\mathbf{Q}}_{h}$ and $C_{\mu}^{h} f=\phi_{S, h} \in M_{h}$ ) the first and the third components of the triple $\left(\mathbf{p}, \phi, \phi_{S}\right)$ (resp. $\left.\left(\mathbf{p}_{h}, \phi_{h}, \phi_{S, h}\right)\right)$ that solves (5.6) (resp. (5.10)) with source $S_{f}=\underline{\nu \Sigma_{f} f}$.

For the DD $+L^{2}$-jumps method, the transposition of Lemma 4.16 reads:

Lemma 6.7. Let $\varphi$ and $\varphi^{\prime}$ be in $W$. Then, it holds:

$$
\begin{aligned}
\left(\underline{\nu \Sigma_{f} \varphi},\left(B_{\mu}-B_{\mu}^{h}\right) \varphi^{\prime}\right)_{0, \mathcal{R}}=a & \left(A_{\mu} \varphi,\left(A_{\mu}-A_{\mu}^{h}\right) \varphi^{\prime}\right)+b\left(\left(A_{\mu}-A_{\mu}^{h}\right) \varphi^{\prime}, B_{\mu} \varphi\right) \\
& +b\left(A_{\mu} \varphi,\left(B_{\mu}-B_{\mu}^{h}\right) \varphi^{\prime}\right)+t\left(B_{\mu} \varphi,\left(B_{\mu}-B_{\mu}^{h}\right) \varphi^{\prime}\right)
\end{aligned}
$$

and

$$
\begin{aligned}
0=a & \left(A_{\mu}^{h} \varphi,\left(A_{\mu}-A_{\mu}^{h}\right) \varphi^{\prime}\right)+b\left(\left(A_{\mu}-A_{\mu}^{h}\right) \varphi^{\prime}, B_{\mu}^{h} \varphi\right) \\
& +b\left(A_{\mu}^{h} \varphi,\left(B_{\mu}-B_{\mu}^{h}\right) \varphi^{\prime}\right)+t\left(B_{\mu}^{h} \varphi,\left(B_{\mu}-B_{\mu}^{h}\right) \varphi^{\prime}\right) .
\end{aligned}
$$

The formulas (6.6) and (4.33), resp. (6.7) and (4.34), are identical. As Strang's Lemma holds for the DD $+L^{2}$ jumps method with nested meshes, we can also transpose Proposition 4.17. For that, we admit that the result of Lemma 6.1 can be improved for smooth functions q. As a matter of fact, in this case one may directly compare the discrete normal traces $\Pi_{f, R}\left(\mathbf{q} \cdot \mathbf{n}_{\mid \Gamma_{f c}}\right)$ and $\Pi_{c, R}\left(\mathbf{q} \cdot \mathbf{n}_{\mid \Gamma_{f c}}\right)$ to the exact normal trace $\mathbf{q} \cdot \mathbf{n}_{\mid \Gamma_{f c}}$, and evaluate the difference in $L^{2}\left(\Gamma_{f c}\right)$-norm, because for smooth functions the exact normal trace always belongs to $L^{2}\left(\Gamma_{f c}\right)$.

Proposition 6.8. For every $\varphi$ in $W$, the following inequalities hold for the $D D+L^{2}$-jumps method with nested meshes:

$$
\begin{aligned}
\left\|\left(B_{\mu}-B_{\mu}^{h}\right) \varphi\right\|_{0, \mathcal{R}} & \lesssim h^{\widetilde{\omega}}\|\varphi\|_{W} ; \\
\left\|\left(A_{\mu}-A_{\mu}^{h}\right) \varphi\right\|_{\mathbf{H}(\operatorname{div}, \mathcal{R})} & \lesssim h^{\widetilde{\omega}}\|\varphi\|_{W} .
\end{aligned}
$$

Estimate (4.37) on the gap between $W$ and $W_{h}$ is still valid: $\hat{\delta}\left(W, W_{h}\right) \lesssim h^{\widetilde{\omega}}$. Let $E_{h}$ be the operator defined in (4.38). We recall that $E_{h}$ and $B_{\mu}^{h}$ commute (Lem. 4.18 holds). The restriction of $E_{h}$ to $W$, denoted by $F_{h}$ is a bijection that satisfies estimate (4.39), for $h$ small enough. We will also make use of $\mathcal{S}_{h}=F_{h}^{-1} E_{h}-I$ that satisfies Lemma 4.20 and Proposition 4.21. We recall that $\hat{B}_{\mu}=\left.B_{\mu}\right|_{W}$ and $\hat{B}_{\mu}^{h}=F_{h}^{-1} B_{\mu}^{h} F_{h}$. The transposition of Theorem 4.22 is stated next. The proof is identical (replace $\omega$ by $\widetilde{\omega}$ ), so it is omitted.

Theorem 6.9. For $h$ small enough, one has for the $D D+L^{2}$-jumps method with nested meshes:

$$
\left\|\hat{B}_{\mu}-\hat{B}_{\mu}^{h}\right\|_{\mathcal{L}(W)} \lesssim h^{2 \widetilde{\omega}}
$$

Corollary 6.10. For $h$ small enough, the error on the eigenvalue for the $D D+L^{2}$-jumps method with nested meshes is given by:

$$
\left|\nu-\nu_{h}\right| \lesssim h^{2 \widetilde{\omega}}
$$




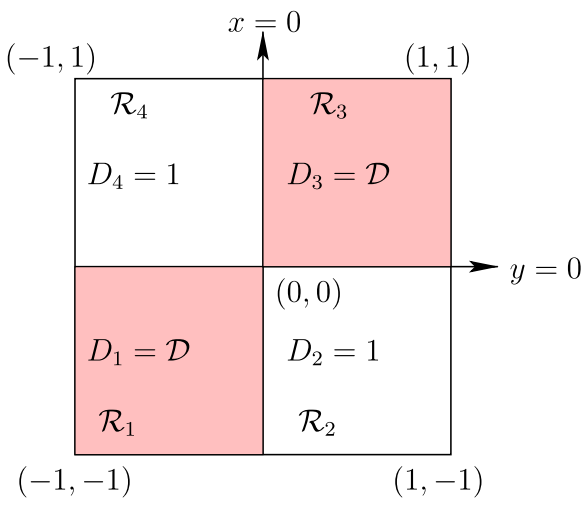

\begin{tabular}{|l|l|l|l|}
\hline$h_{c}$ & $h_{c}$ & $h_{c}$ & $h_{c}$ \\
\hline$h_{c}$ & $h_{f}$ & $h_{f}$ & $h_{c}$ \\
\hline$h_{c}$ & $h_{f}$ & $h_{f}$ & $h_{c}$ \\
\hline$h_{c}$ & $h_{c}$ & $h_{c}$ & $h_{c}$ \\
\hline
\end{tabular}

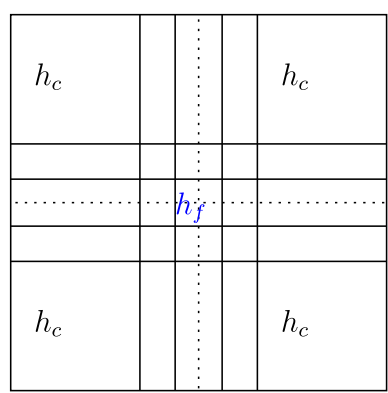

Figure 2. The domain of study, and the subdomain meshsizes.

\subsection{About non-nested meshes}

We recall that, for general non-nested meshes, one has convergence without explicit convergence rate, as soon as (5.11)-(5.12) hold uniformly. In the most general case however, it seems difficult to obtain a convergence error that depends explicitly on $h$.

On the other hand, let us consider the case where the meshes are non-nested, with some structure. By structure, it is understood that the non-nestedness can be described by a finite number of configurations (e.g. 3 -face mesh vs. 5 -face mesh, etc.) that are reproduced at smaller and smaller scales when the meshsize diminishes.

We note first that a result similar to Lemma 6.1 can be recovered. Going back to the reference configurations (by assumption there are a finite number of them) and taking the supremum in the upper bounds among all these configurations, we infer from (A.5) that $\left\|\left[\tilde{\mathbf{q}}_{R} \cdot \mathbf{n}\right]\right\|_{0, \Gamma_{f c}} \lesssim h_{c \mid \Gamma_{f c}}\left\|q_{f, h}\right\|_{0, \Gamma_{f c}}$, i.e. one can conclude the proof as before. As a consequence, an explicit convergence rate may be derived for the source problem as in Theorem 6.2.

Then, one may proceed in a similar fashion to prove Lemma 6.4, so as to derive an Aubin-Nitsche estimate as in Theorem 6.5. Finally, because interface terms are absent in the analysis of the convergence rate of the eigenvalues (see in particular (6.6)-(6.7)), such estimates can also be proved for non-nested meshes, with some structure.

\section{NUMERICAL ILLUSTRATIONS}

The tests are carried out in two dimensions: the cartesian coordinates are denoted by $(x, y)$. We use $\mathrm{RTN}_{[0]}$ finite elements on rectangular meshes. We define the discrete space of Lagrange multipliers $M_{h}$ as in (5.13).

\subsection{Benchmark square for transmission problems}

We study a singular toy problem described on Dauge's website [15] for a magnetic problem and adapted here for the neutron diffusion equation with Neuman boundary condition. Set $\mathcal{R}:=]-1,1\left[{ }^{2}\right.$, and divide it into four subsquares (see Fig. 2 left). Let $D$, be a scalar, piecewise-constant, coefficient: $D:=\mathcal{D}=0.1$ in $\mathcal{R}_{1} \cup \mathcal{R}_{3}$, and 1 elsewhere, $\Sigma_{a}=1$ and $\underline{\nu \Sigma_{f}}=1$. We consider the following problem:

$$
\begin{cases}-\operatorname{div} D \operatorname{grad} \phi+\phi=\lambda \phi & \text { in } \mathcal{R} \\ \frac{\partial \phi}{\partial n}=0 & \text { on } \partial \mathcal{R} .\end{cases}
$$

The singularity exponent is $r_{\max } \approx 0.39$. Implementation is in MATLAB. 
TABLE 1. Results with 16 subdomains.

\begin{tabular}{cccccc}
\hline $1 / h$ & $N_{\phi}$ & $\varepsilon_{\lambda_{1}}$ & $\varepsilon_{\lambda_{2}}$ & $\varepsilon_{\lambda_{3}}$ & $\varepsilon_{\lambda_{4}}$ \\
\hline 4 & 448 & $2.88 e-3$ & $3.92 e-2$ & $5.49 e-3$ & $2.00 e-2$ \\
8 & 1792 & $7.22 e-4$ & $2.36 e-2$ & $1.38 e-3$ & $5.00 e-3$ \\
12 & 4032 & $3.22 e-4$ & $1.74 e-2$ & $6.12 e-4$ & $2.22 e-3$ \\
16 & 7168 & $1.81 e-4$ & $1.40 e-2$ & $3.44 e-4$ & $1.25 e-3$ \\
20 & 11200 & $1.16 e-4$ & $1.18 e-2$ & $2.20 e-4$ & $8.00 e-4$ \\
24 & 16128 & $8.05 e-5$ & $1.02 e-2$ & $1.53 e-4$ & $5.05 e-4$ \\
\multicolumn{2}{c}{ Rate } & $h^{2}$ & $h^{0.76}$ & $h^{2}$ & $h^{2}$ \\
\hline \multicolumn{7}{c}{}
\end{tabular}

TABLE 2. Results with 25 subdomains using graded meshes.

\begin{tabular}{cccccc}
\hline $1 / h$ & $N_{\phi}$ & $\varepsilon_{\lambda_{1}}$ & $\varepsilon_{\lambda_{2}}$ & $\varepsilon_{\lambda_{3}}$ & $\varepsilon_{\lambda_{4}}$ \\
\hline 3 & 304 & $7.47 e-3$ & $1.14 e-2$ & $1.92 e-2$ & $1.12 e-1$ \\
6 & 1216 & $1.92 e-3$ & $8.19 e-3$ & $4.90 e-3$ & $2.75, e-2$ \\
12 & 4864 & $4.83 e-4$ & $5.28 e-3$ & $1.23 e-3$ & $6.85 e-3$ \\
15 & 7600 & $3.10 e-4$ & $4.42 e-3$ & $7.88 e-4$ & $4.38 e-3$ \\
18 & 10944 & $2.15 e-4$ & $3.86 e-3$ & $5.47 e-4$ & $3.04 e-3$ \\
21 & 14896 & $1.59 e-4$ & $6.68 e-4$ & $4.02 e-4$ & $2.24 e-3$ \\
\multicolumn{2}{c}{ Rate } & $h^{2}$ & $h^{0.71}$ & $h^{2}$ & $h^{2}$ \\
\hline \multicolumn{7}{c}{} \\
\hline
\end{tabular}

We study the error on the four first eigenvalues (excluding $\lambda_{0}=1$ ), with two different partitions $\left\{\widetilde{\mathcal{R}}_{i}\right\}_{1 \leq i \leq \widetilde{N}}$. The results are given in Tables 1 and 2, which data are:

- $h$ : the meshsize,

- $N_{\phi}$ : the number of degrees of freedom of $\phi$,

- $\varepsilon_{\lambda_{i}}=\left|\lambda_{h, i}-\lambda_{i}\right| /\left|\lambda_{i}\right|$ : the relative error for the eigenvalue $\lambda_{i}, i=1,4$.

In the last line, we report the average rate of convergence of the computations. In Figure 3a (resp. 3b), we represented the mesh for $1 / h=12$ (resp. $1 / h=18$ ) and the second non-constant eigenfunction $\phi_{2}$, which is singular at the cross-point.

The first partition is based on $\widetilde{N}=16$ square subdomains, represented in Figure 2 middle. As indicated in this figure, the four centered subdomains have a meshsize equal to $h_{f}$ whereas the other subdomains have a meshsize equal to $h_{c}=2 h_{f}$, so that the parameter is $h=h_{c}$. The results are given in Table 1 .

The second partition is based on $\widetilde{N}=25$ subdomains, with graded meshes towards the cross-point, where the singular behaviour is expected. The subdomain in the center of $\mathcal{R}$ has a mesh size equal to $h_{f}$, whereas the four subdomains on the corners of $\mathcal{R}$ have a meshsize equal to $h_{c}=6 h_{f}$ (see Fig. 2 right). This is similar in spirit to the XFEM except there is only one mesh near the cross-point [20].

The results are given in Table 2. With this simple idea (the use of graded meshes), one derives an accurate approximation of the singular eigenfunction at low cost. Indeed, comparing Tables 1 and 2, one notices that the error $\varepsilon_{\lambda_{2}}$ is comparable using the coarser mesh of the second partition (with $N_{\phi}=304$ ) than using the finer mesh of the first partition (with $N_{\phi}=16128$ ). However, the approximation of eigenvalues associated to smooth eigenfunctions is not improved by the use of graded meshes. On the contrary, as the order of the eigenvalues increases, their approximations seem to be more and more degraded, which is due to the difficulty to capture the faster and faster oscillations of the corresponding eigenfunctions. 


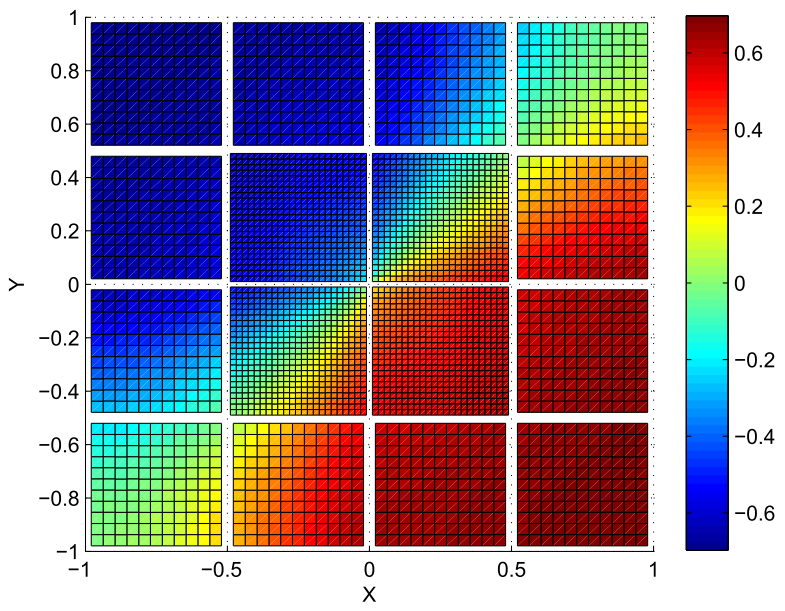

(a) $\phi_{2}, 16$ subdomains

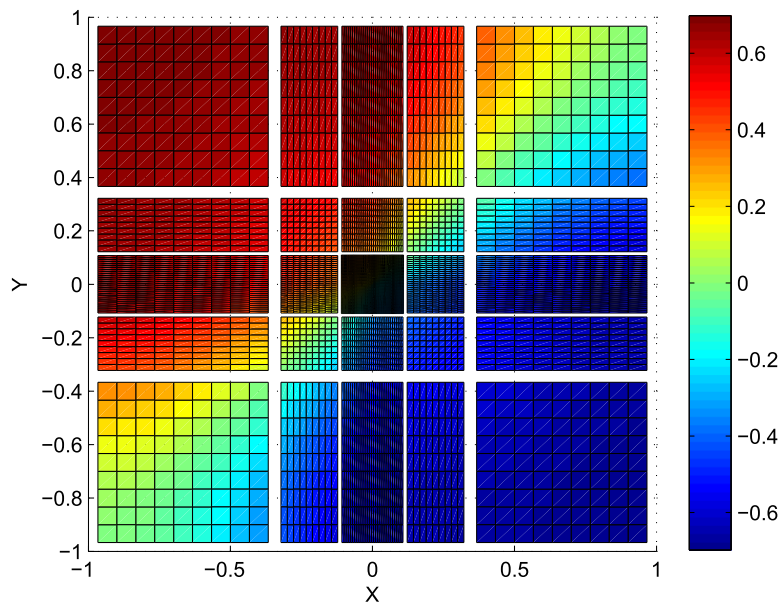

(b) $\phi_{2}, 25$ subdomains

FiguRE 3. The second non-constant eigenfunction.

\subsection{PWR core}

We give here some results of computations carried out with the MINOS solver of the APOLLO3 ${ }^{\circledR 5}$ neutronics code [30] developed at CEA. This industrial test models a pressurized water large reactor core with heavy-steel reflector similar to the one described in [29]. The neutron transport equation is discretized using the multigroup simplified $P_{N}\left(S P_{N}\right)$ equations, with two groups of energy, and $S P_{1}$ and $S P_{3}$ angular orders. We recall that, for each group, the neutron $S P_{1}$ equation is similar to the neutron diffusion equation, whereas the neutron $S P_{3}$ equation corresponds to two coupled neutron diffusion equations. The different homogenization steps that allow to obtain the coefficients of this discretization on square cells lead to 229 different media. The coefficients are thus parametrized according to the medium, the energy group and the angular order, which depend respectively on the position, the energy and the direction of the neutrons. We refer to $[23,24,25]$ for more details on the multigroup $S P_{N}$ and diffusion neutron equations and the general algorithm to solve them.

The subdomains $\left\{\widetilde{\mathcal{R}}_{i}\right\}_{1 \leq i \leq 361}$ of the partition correspond to the $19 \times 19$ cells of Figure 1a. In each subdomain, the coarser triangulation is also such that the coefficients are piecewise constant. The meshes of the subdomains are nested.

In neutronics, the quantity of interest is the inverse of the smallest eigenvalue, which is called the criticality, and is denoted by $k_{\text {eff. }}$. Below, we make comparisons on the criticality, the reference value, denoted by $k_{\text {eff }}^{\text {ref }}$, being computed on a conforming mesh made of $1.5 e+7$ (resp. $7.5 e+6)$ rectangles in $S P_{1}$ (resp. $S P_{3}$ ).

In Table 3, we present the results obtained with the MINOS solver for different levels of refinement, with $\mathrm{RTN}_{[0]}$ finite elements on rectangles. The data are:

- $h$ : the meshsize,

- $N_{\phi}$ : the spatial number of degrees of freedom of the neutron flux $\phi$,

- $\varepsilon_{1}$ (resp. $\left.\varepsilon_{3}\right)$ : the relative error made on the criticality $\left|k_{\text {eff }}-k_{\text {eff }}^{r e f}\right| / k_{\text {eff }}^{r e f}$, for a computation using the $S P_{1}$ (resp. $S P_{3}$ ) approximation.

- rate: the averaged rate of convergence.

Convergence rates are higher than 1, seemingly indicating the absence of strong singularities in the first eigenfunction. Instead, we hypothetize that we are still in the pre-asymptotic regime (for the first eigenfunction):

\footnotetext{
${ }^{5}$ APOLLO3 is a registered trademark in France.
} 
TABLE 3. Results with 361 subdomains.

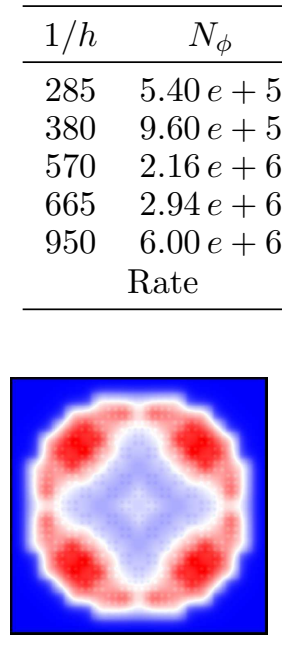

(a) First group

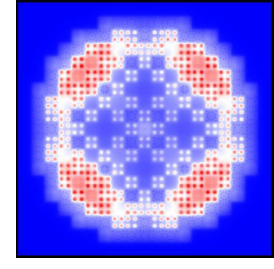

(b) Second group

Figure 4. Neutron flux.

on the one hand, the norm of the "more singular" part is small compared to the norm of the "more regular" part, and on the other hand there are only a few degrees of freedom per characteristic length (see Fig. 1b).

Note that the DD version is parallelized in the APOLLO3 ${ }^{\circledR}$ code, contrary to the plain version. Hence, computational times are greatly reduced: we refer to [23] for the analyses of algorithms and their parallelization.

The neutron flux of the first (resp. second) group of energy are represented in Figure 4a (resp. Fig. 4b).

\section{Conclusion}

The solution of the steady-state one-group neutron diffusion equation being usually of low-regularity, the convergence of the eigenvalues and the error estimates are not straightforward to obtain. In particular, we provide new proofs:

- for the source and eigen-problems, with low-regularity solutions;

- for the eigenproblems, in mixed setting with non-vanishing zero-order term $\left(\Sigma_{a} \neq 0\right)$.

Notice that our results are obtained under the regular ${ }^{+}$condition on the family of triangulations. For the DD case, we suggest the following strategies to take into account the apparently restrictive condition on quasiuniform meshes on the interface, compared to the regular ${ }^{+}$condition on the family of triangulations:

- use $\left\{\widetilde{\mathcal{R}}_{i}\right\}_{1 \leq i \leq \widetilde{N}}$ for DD as the orthogonal (i.e. Voronoï) tessellation of $\left\{\mathcal{R}_{i}\right\}_{1 \leq i \leq N}$;

- use $\left\{\widetilde{\mathcal{R}}_{i}\right\}_{1<i<\widetilde{N}}=\left\{\mathcal{R}_{i}\right\}_{1 \leq i \leq N}$ and compute the singular part of the solution (or eigenfunction) via some ad hoc technique (SCM, XFEM, etc.).

A possible continuation of this paper is the study of the steady-state multigroup neutron $S P_{N}$ problem [21].

\section{Appendix A. Additional proofs}

We provide here the proof of three technical lemmas.

Let $\left(\mathcal{T}_{h}\right)_{h}$ be a given regular family of triangulations. We call $\hat{K}:=[0,1]^{d}$ the reference element. Let $h$ be given. For every $K \in \mathcal{T}_{h}$, we denote by $\mathbf{x}=F_{K}(\hat{\mathbf{x}}):=\mathbb{A}_{K} \hat{\mathbf{x}}+\mathbf{b}_{K}, \mathbb{A}_{K} \in \mathbb{R}^{d \times d}, \mathbf{b}_{K} \in \mathbb{R}^{d}$, the map from $\hat{K}$ to 
$K$. Introducing $h_{K}=\operatorname{diam}(K)$ for all $K \in \mathcal{T}_{h}$, one may bound $\left\|\mathbb{A}_{K}\right\|,\left\|\left(\mathbb{A}_{K}\right)^{-1}\right\|,\left|\operatorname{det}\left(\mathbb{A}_{K}\right)\right|$ with respect to $h_{K}$. The change of variable formulas from $\hat{K}$ to $K$, and vice versa, can be found e.g. in Section 1 of [17].

Proof of Lemma 4.13. We follow Section 2 of [2]. Given $\psi_{h} \in L_{h}^{k}$, one has $\psi_{h} \in H^{\mu}(\mathcal{R})$, for all $\mu<1 / 2$. By the definition of the norm of $H^{\mu}(\mathcal{R})$, we have the following equalities:

$$
\begin{aligned}
\left\|\psi_{h}\right\|_{\mu, \mathcal{R}}^{2} & =\left\|\psi_{h}\right\|_{0, \mathcal{R}}^{2}+\int_{\mathcal{R}} \int_{\mathcal{R}} \frac{\left|\psi_{h}(\mathbf{x})-\psi_{h}(\mathbf{y})\right|^{2}}{|\mathbf{x}-\mathbf{y}|^{d+2 \mu}} \mathrm{d} \mathbf{y} \mathrm{d} \mathbf{x} \\
& =\sum_{K \in \mathcal{T}_{h}}\left(\left\|\psi_{h}\right\|_{0, K}^{2}+\int_{K} \int_{\mathcal{R}} \frac{\left|\psi_{h}(\mathbf{x})-\psi_{h}(\mathbf{y})\right|^{2}}{|\mathbf{x}-\mathbf{y}|^{d+2 \mu}} \mathrm{d} \mathbf{y} \mathrm{d} \mathbf{x}\right) \\
& =\sum_{K \in \mathcal{T}_{h}}\left\|\psi_{h}\right\|_{\mu, K}^{2}+\sum_{K \in \mathcal{T}_{h}} \int_{K} \int_{\mathcal{R} \backslash K} \frac{\left|\psi_{h}(\mathbf{x})-\psi_{h}(\mathbf{y})\right|^{2}}{|\mathbf{x}-\mathbf{y}|^{d+2 \mu}} \mathrm{d} \mathbf{y} \mathrm{d} \mathbf{x} .
\end{aligned}
$$

Let us estimate first $\sum_{K \in \mathcal{T}_{h}}\left\|\psi_{h}\right\|_{\mu, K}^{2}$. According to Corollary 1.138 of [17], we know that

$$
\sum_{K \in \mathcal{T}_{h}}\left\|\psi_{h}\right\|_{\mu, K}^{2} \lesssim \sum_{K \in \mathcal{T}_{h}} h_{K}^{-2 \mu}\left\|\psi_{h}\right\|_{0, K}^{2} \lesssim h_{\min }^{-2 \mu}\left\|\psi_{h}\right\|_{0, \mathcal{R}}^{2}
$$

where $h_{\min }=\min _{K \in \mathcal{T}_{h}} h_{K}$. To estimate the remaining part, we recall that, for any $K \in \mathcal{T}_{h}$ and any $\mathbf{x} \in K$, it holds that, by going back the reference space, applying (cf. [22], Eq. (1.3.2.12)) on $\hat{K}$ and then going to the physical space:

$$
\int_{\mathcal{R} \backslash K} \frac{1}{|\mathbf{x}-\mathbf{y}|^{d+2 \mu}} \mathrm{d} \mathbf{y} \lesssim \frac{1}{\rho_{\partial K}(\mathbf{x})^{2 \mu}},
$$

where $\rho_{\partial K}(\mathbf{x})=\inf _{\mathbf{y} \in \partial K}|\mathbf{x}-\mathbf{y}|$. Thus we have:

$$
\begin{aligned}
\int_{K} \int_{\mathcal{R} \backslash K} \frac{\left|\psi_{h}(\mathbf{x})-\psi_{h}(\mathbf{y})\right|^{2}}{|\mathbf{x}-\mathbf{y}|^{d+2 \mu}} \mathrm{d} \mathbf{y} \mathrm{d} \mathbf{x} & =\sum_{\substack{K^{\prime} \in \mathcal{T}_{h} \\
K^{\prime} \neq K}} \int_{K} \int_{K^{\prime}} \frac{\left|\psi_{h}(\mathbf{x})-\psi_{h}(\mathbf{y})\right|^{2}}{|\mathbf{x}-\mathbf{y}|^{d+2 \mu}} \mathrm{d} \mathbf{y} \mathrm{d} \mathbf{x} \\
& \lesssim \sum_{\substack{K^{\prime} \in \mathcal{T}_{h} \\
K^{\prime} \neq K}} \int_{K} \int_{K^{\prime}} \frac{\psi_{h}(\mathbf{x})^{2}+\psi_{h}(\mathbf{y})^{2}}{|\mathbf{x}-\mathbf{y}|^{d+2 \mu}} \mathrm{d} \mathbf{y} \mathrm{d} \mathbf{x} \\
& \lesssim \int_{K} \frac{\psi_{h}(\mathbf{x})^{2}}{\rho_{\partial K}(\mathbf{x})^{2 \mu}} \mathrm{d} \mathbf{x} .
\end{aligned}
$$

Going back to the reference element $\hat{K}$ and introducing $\psi_{h \mid K}(\mathbf{x})=\hat{\psi}(\hat{\mathbf{x}})$, it stands:

$$
\int_{K} \frac{\psi_{h}(\mathbf{x})^{2}}{\rho_{\partial K}(\mathbf{x})^{2 \mu}} \mathrm{d} \mathbf{x} \lesssim h_{K}^{d-2 \mu} \int_{\hat{K}} \frac{\hat{\psi}(\hat{\mathbf{x}})^{2}}{\rho_{\partial \hat{K}}(\hat{\mathbf{x}})^{2 \mu}} \mathrm{d} \hat{\mathbf{x}} .
$$

Because $\mu<1 / 2$ (cf. [22], Thm. 1.4.4.4), $\hat{\psi} \mapsto\left(\int_{\hat{K}} \hat{\psi}(\hat{\mathbf{x}})^{2} \rho_{\partial \hat{K}}(\hat{\mathbf{x}})^{-2 \mu} \mathrm{d} \hat{\mathbf{x}}\right)^{1 / 2}$ is a norm on $\hat{L}^{k}=Q_{k, k, k}(\hat{K})$. Thanks to the equivalence of the norms on finite dimensional vector spaces, one gets

$$
\int_{K} \frac{\psi_{h}(\mathbf{x})^{2}}{\rho_{\partial K}(\mathbf{x})^{2 \mu}} \mathrm{d} \mathbf{x} \lesssim h_{K}^{d-2 \mu}\|\hat{\psi}\|_{0, \hat{K}}^{2}
$$


Finally, going back to element $K$, we know that $\|\hat{\psi}\|_{0, \hat{K}}^{2} \lesssim h_{K}^{-d}\left\|\psi_{h}\right\|_{0, K}^{2}$. Hence using (A.3) and the results that follow, we have:

$$
\int_{K} \int_{\mathcal{R} \backslash K} \frac{\left|\psi_{h}(\mathbf{x})-\psi_{h}(\mathbf{y})\right|^{2}}{|\mathbf{x}-\mathbf{y}|^{d+2 \mu}} \mathrm{d} \mathbf{y} \mathrm{d} \mathbf{x} \lesssim h_{K}^{-2 \mu}\left\|\psi_{h}\right\|_{0, K}^{2}
$$

Starting from (A.1) using (A.2) and (A.4), we obtain finally the global bound:

$$
\left\|\psi_{h}\right\|_{\mu, \mathcal{R}} \lesssim h_{\min }^{-\mu}\left\|\psi_{h}\right\|_{0, \mathcal{R}}
$$

As the family of triangulations is regular ${ }^{+}$, one has $h_{\min }^{-\mu} \lesssim h^{(\theta-2) \mu}$, which concludes the proof.

Proof of Lemma 6.1. For $l=c, f$, we introduce the operators from the normal trace spaces $(\mathbf{H}(\operatorname{div}, \mathcal{R}) \cap$ $\left.\mathbf{H}^{\mu}(\mathcal{R})\right) \cdot \mathbf{n}_{\mid \Gamma_{f c}}$ to the discrete spaces of normal traces $T_{l, h}$ on $\Gamma_{f c}$ :

$$
\left\{\begin{array}{l}
\Pi_{l, R}:\left(\mathbf{H}(\operatorname{div}, \mathcal{R}) \cap \mathbf{H}^{\mu}(\mathcal{R})\right) \cdot \mathbf{n}_{\mid \Gamma_{f c}} \rightarrow T_{l, h \mid \Gamma_{f c}} \\
\mathbf{q}^{\prime} \cdot \mathbf{n}_{\mid \Gamma_{f c}} \mapsto \tilde{\mathbf{q}}_{l, R}^{\prime} \cdot \mathbf{n}_{\mid \Gamma_{f c}} .
\end{array}\right.
$$

With a slight abuse of notations, we write $\Pi_{l, R}\left(\mathbf{q}_{l}^{\prime} \cdot \mathbf{n}_{\mid \partial \widetilde{\mathcal{R}}_{l}}\right)=\tilde{\mathbf{q}}_{l, R}^{\prime} \cdot \mathbf{n}_{\mid \partial \widetilde{\mathcal{R}}_{l}} \cdot$ We also introduce the operator $\Pi_{c, R}^{0}$ on the vector space of normal traces of elements of $\widetilde{\mathbf{Q}}_{c, h}$ with lowest-order RTN finite element, i.e. the vector space $T_{c, h \mid \Gamma_{f c}}^{0}$ of piecewise constant functions on the interface mesh defined as the trace on $\Gamma_{f c}$ of the mesh used in $\widetilde{\mathcal{R}}_{c}$. Note that because the meshes are nested, the restriction of $\Pi_{f, R}$ (resp., $\Pi_{c, R}$ and $\Pi_{c, R}^{0}$ ) on $T_{f, h \mid \Gamma_{f c}}$ (resp., on the subspaces $T_{c, h \mid \Gamma_{f c}}$ and $T_{c, h \mid \Gamma_{f c}}^{0}$ where applicable) may also be considered as an orthogonal projection operator. Denoting $q_{f, h}=\Pi_{f, R}\left(\mathbf{q} \cdot \mathbf{n}_{\mid \Gamma_{f c}}\right)$, we have:

$$
\begin{aligned}
\left\|\left[\tilde{\mathbf{q}}_{R} \cdot \mathbf{n}\right]\right\|_{0, \Gamma_{f c}} & =\left\|\Pi_{f, R}\left(\mathbf{q} \cdot \mathbf{n}_{\mid \Gamma_{f c}}\right)-\Pi_{c, R}\left(\mathbf{q} \cdot \mathbf{n}_{\mid \Gamma_{f c}}\right)\right\|_{0, \Gamma_{f c}} \\
& =\left\|\Pi_{f, R}\left(\mathbf{q} \cdot \mathbf{n}_{\mid \Gamma_{f c}}\right)-\Pi_{c, R} \circ \Pi_{f, R}\left(\mathbf{q} \cdot \mathbf{n}_{\mid \Gamma_{f c}}\right)\right\|_{0, \Gamma_{f c}} \\
& =\left\|\left(\mathbb{I}-\Pi_{c, R}\right) q_{f, h}\right\|_{0, \Gamma_{f c}} \\
& \leq\left\|\left(\mathbb{I}-\Pi_{c, R}^{0}\right) q_{f, h}\right\|_{0, \Gamma_{f c}} .
\end{aligned}
$$

As the meshes are quasi-uniform on the interface, one has $h_{c \mid \Gamma_{f c}} \approx h_{f \mid \Gamma_{f c}}$. Then, starting from (A.5), thanks to the quasi-uniform mesh assumption for the inverse inequalities on $\Gamma_{f c}, c f$. Lemma 10.10 of [31], we find

$$
\begin{aligned}
\left\|\left[\tilde{\mathbf{q}}_{R} \cdot \mathbf{n}\right]\right\|_{0, \Gamma_{f c}} & \lesssim h_{c \mid \Gamma_{f c}}\left\|q_{f, h}\right\|_{0, \Gamma_{f c}}([3], \text { Lem. 4.9) } \\
& \lesssim h_{c \mid \Gamma_{f c}}\left(h_{f \mid \Gamma_{f c}}\right)^{-1 / 4}\left\|q_{f, h}\right\|_{-1 / 4, \Gamma_{f c}} \\
& \lesssim\left(h_{f \mid \Gamma_{f c}}\right)^{3 / 4}\left\|\Pi_{f, R}\left(\mathbf{q} \cdot \mathbf{n}_{\mid \partial \widetilde{\mathcal{R}}_{f}}\right)\right\|_{-1 / 4, \partial \widetilde{\mathcal{R}}_{f}} \\
& \lesssim\left(h_{f \mid \Gamma_{f c}}\right)^{3 / 4}\left(h_{f \mid \partial \widetilde{\mathcal{R}}_{f}}\right)^{-1 / 4}\left\|\tilde{\mathbf{q}}_{f, R} \cdot \mathbf{n}_{\mid \partial \widetilde{\mathcal{R}}_{f}}\right\|_{-1 / 2, \partial \widetilde{\mathcal{R}}_{f}} \\
& \lesssim h_{f}^{1 / 2}\left\|\tilde{\mathbf{q}}_{f, R}\right\|_{\mathbf{H}\left(\operatorname{div}, \widetilde{\mathcal{R}}_{f}\right)} \lesssim h_{f}^{1 / 2}\left\|\mathbf{q}_{f}\right\|_{\mathbf{H}\left(\operatorname{div}, \widetilde{\mathcal{R}}_{f}\right)} .
\end{aligned}
$$

Above, we have used the continuity of the normal trace, resp. the stability of the RTN interpolant, to derive the last two inequalities.

Proof of Lemma 6.4. First, let us bound the norm of $\left\|\delta \mathbf{q}_{f c}\right\|_{\mathbf{H}\left(\operatorname{div}, \widetilde{\mathcal{R}}_{f}\right)}$ by $\left\|\delta \mathbf{q}_{f c} \cdot \mathbf{n}\right\|_{0, \Gamma_{f c}}$. We use the notation $\mathbf{v}=\delta \mathbf{q}_{f c}$ below. Denoting by $\left(K_{\ell}\right)_{\ell}$ the parallelepipeds composing the mesh on $\widetilde{\mathcal{R}}_{f}$, and $\mathcal{N}_{\Gamma}$ the set of indices $\ell$ 
such that $\Gamma_{\ell}:=K_{\ell} \cap \Gamma_{f c}$ is of Hausdorff dimension $d-1$, because of the definition of $\mathbf{v}$ it now holds

$$
\|\mathbf{v}\|_{\mathbf{H}\left(\operatorname{div}, \widetilde{\mathcal{R}}_{f}\right)}^{2}=\sum_{\ell}\left\|\mathbf{v}_{\mid K_{\ell}}\right\|_{\mathbf{H}\left(\operatorname{div}, K_{\ell}\right)}^{2}=\sum_{\ell \in \mathcal{N}_{\Gamma}}\left\|\mathbf{v}_{\mid K_{\ell}}\right\|_{\mathbf{H}\left(\operatorname{div}, K_{\ell}\right)}^{2} .
$$

Then, one can bound $\left\|\mathbf{v}_{\mid K_{\ell}}\right\|_{\mathbf{H}\left(\operatorname{div}, K_{\ell}\right)}$ by $\left\|\mathbf{v}_{\mid K_{\ell}} \cdot \mathbf{n}\right\|_{0, \Gamma_{\ell}}$ for each index $\ell \in \mathcal{N}_{\Gamma}$. To that aim, one goes back to the reference element $\hat{K}$ via the Piola transform, which reads ([7], Sect. 2.1.3):

$$
\mathbf{v}_{\mid K_{\ell}}(\mathbf{x})=\frac{1}{\left|\operatorname{det}\left(\mathbb{A}_{K_{\ell}}\right)\right|} \mathbb{A}_{K_{\ell}} \hat{\mathbf{v}}(\hat{\mathbf{x}}), \quad \operatorname{div} \mathbf{v}_{\mid K_{\ell}}(\mathbf{x})=\frac{1}{\left|\operatorname{det}\left(\mathbb{A}_{K_{\ell}}\right)\right|} \operatorname{div} \hat{\mathbf{v}}(\hat{\mathbf{x}})
$$

With the help of a classical formula for the change of variables on $\Gamma_{\ell}$ ([7], Eq. (2.1.62)), one finds after a few elementary algebraic manipulations ${ }^{6}$ that

$$
h_{K_{\ell}}^{d-1} \int_{\Gamma_{\ell}}\left(\mathbf{v}_{\mid K_{\ell}} \cdot \mathbf{n}\right)^{2} \mathrm{~d} \Gamma \approx \int_{\hat{\Gamma}_{\ell}}(\hat{\mathbf{v}} \cdot \hat{\mathbf{n}})^{2} \mathrm{~d} \hat{\Gamma}
$$

where $\hat{\Gamma}_{\ell}$ is equal to $F_{K_{\ell}}^{-1}\left(\Gamma_{\ell}\right)$.

On the reference element, it holds

$$
\|\hat{\mathbf{v}}\|_{\mathbf{H}(\operatorname{div}, \hat{K})}^{2} \lesssim \int_{\hat{\Gamma_{\ell}}}(\hat{\mathbf{v}} \cdot \hat{\mathbf{n}})^{2} \mathrm{~d} \hat{\Gamma}
$$

because the non-zero degrees of freedom are all located on $\hat{\Gamma}_{\ell}$. Finally, one has the classical bounds ([7], Lem. 2.1.7):

$$
\left\|\mathbf{v}_{\mid K_{\ell}}\right\|_{0, K_{\ell}}^{2} \lesssim h_{K_{\ell}}^{2-d}\|\hat{\mathbf{v}}\|_{0, \hat{K}}^{2} \quad\left\|\operatorname{div} \mathbf{v}_{\mid K_{\ell}}\right\|_{0, K_{\ell}}^{2} \lesssim h_{K_{\ell}}^{-d}\|\operatorname{div} \hat{\mathbf{v}}\|_{0, \hat{K}}^{2}
$$

so that

$$
\left\|\mathbf{v}_{\mid K_{\ell}}\right\|_{\mathbf{H}\left(\operatorname{div}, K_{\ell}\right)}^{2} \lesssim h_{K_{\ell}}^{-d}\|\hat{\mathbf{v}}\|_{\mathbf{H}(\hat{\mathrm{div}}, \hat{K})}^{2} \lesssim h_{K_{\ell}}^{-1} \int_{\Gamma_{\ell}}\left(\mathbf{v}_{\mid K_{\ell}} \cdot \mathbf{n}\right)^{2} \mathrm{~d} \Gamma
$$

Adding up the contributions for $\ell \in \mathcal{N}_{\Gamma}$, one finds:

$$
\left\|\delta \mathbf{q}_{f c}\right\|_{\mathbf{H}\left(\operatorname{div}, \widetilde{\mathcal{R}}_{f}\right)} \lesssim h_{f}^{-1 / 2}\left\|\delta \mathbf{q}_{f c} \cdot \mathbf{n}\right\|_{0, \Gamma_{f c}} .
$$

By modifying the final computations in the proof of Lemma 6.1, one finds that for all $0<\epsilon<\mu$ :

$$
\begin{aligned}
\left\|\delta \mathbf{q}_{f c} \cdot \mathbf{n}\right\|_{0, \Gamma_{f c}} & \lesssim h_{c \mid \Gamma_{f c}}\left\|q_{f, h}\right\|_{0, \Gamma_{f c}}([3], \text { Lem. 4.9) } \\
& \lesssim h_{c \mid \Gamma_{f c}}\left(h_{f \mid \Gamma_{f c}}\right)^{\epsilon-1 / 2}\left\|q_{f, h}\right\|_{\epsilon-1 / 2, \Gamma_{f c}}([31], \text { Lem. 10.10) } \\
& \lesssim h_{f}^{\epsilon+1 / 2}\left\|q_{f, h}\right\|_{\epsilon-1 / 2, \Gamma_{f c}} \\
& \lesssim h_{f}^{\epsilon+1 / 2}\left\|\Pi_{f, R}\left(\mathbf{q}_{f} \cdot \mathbf{n}_{\mid \partial \widetilde{\mathcal{R}}_{f}}\right)\right\|_{\epsilon-1 / 2, \partial \widetilde{\mathcal{R}}_{f}} \\
& \lesssim h_{f}^{\epsilon+1 / 2}\left\|\mathbf{q}_{f} \cdot \mathbf{n}_{\mid \partial \widetilde{\mathcal{R}}_{f}}\right\|_{\epsilon-1 / 2, \partial \widetilde{\mathcal{R}}_{f}}([2], \text { Thm. 2.4 and Rem. 2.5) } \\
& \lesssim h_{f}^{\epsilon+1 / 2}\left(\left\|\mathbf{q}_{f}\right\|_{\epsilon, \widetilde{\mathcal{R}}_{f}}+\left\|\operatorname{div} \mathbf{q}_{f}\right\|_{0, \widetilde{\mathcal{R}}_{f}}\right) .
\end{aligned}
$$

\footnotetext{
${ }^{6}$ Since the meshes are quasi-uniform on $\Gamma_{f c}$, they are in particular regular.
} 
Or, choosing $\epsilon=\mu-\eta$ for $\eta>0$ arbitrary small, that

$$
\left\|\delta \mathbf{q}_{f c} \cdot \mathbf{n}\right\|_{0, \Gamma_{f c}} \lesssim h_{f}^{\mu+1 / 2-\eta}\left(\left\|\mathbf{q}_{f}\right\|_{\mu, \widetilde{\mathcal{R}}_{f}}+\left\|\operatorname{div} \mathbf{q}_{f}\right\|_{0, \widetilde{\mathcal{R}}_{f}}\right) .
$$

Using (A.6), we conclude the proof.

Acknowledgements. L. Giret and E. Jamelot gratefully acknowledge EDF for its long term partnership and its support.

\section{REFERENCES}

[1] I. Babuska and J. Osborn, Eigenvalue problems, in Vol. II of Handbook of Numerical Analysis, edited by P.G. Ciarlet and J.-L. Lions. North Holland (1991) 641-787.

[2] F. Ben Belgacem and S. Brenner, Some nonstandard finite element estimates with applications to 3D Poisson and Signorini problems. Electron. Trans. Numer. Anal. 12 (2001) 134-148.

[3] A. Bermudez, P. Gamallo, M.R. Nogueiras and R. Rodriguez, Approximation of a structural acoustic vibration problem by hexahedral finite elements. IMA J. Numer. Anal. 26 (2006) 391-421.

[4] D. Boffi, Finite element approximation of eigenvalue problems. Acta Numer. 19 (2010) 1-120.

[5] D. Boffi, F. Brezzi and L. Gastaldi, On the convergence of eigenvalues for mixed formulations. Ann. Scuola Norm. Sup. Pisa Cl. Sci. Ser. 425 (1997) 131-154.

[6] D. Boffi, F. Brezzi and L. Gastaldi, On the problem of spurious eigenvalues in the approximation of linear elliptic problems in mixed form. Math. Comput. 69 (2000) 121-140.

[7] D. Boffi, F. Brezzi and M. Fortin, Mixed and Hybrid Finite Element Methods and Applications. Springer-Verlag (2013).

[8] D. Boffi, D. Gallistl, F. Gardini and L. Gastaldi, Optimal convergence of adaptive FEM for eigenvalue clusters in mixed form. Math. Comput. 86 (2017) 2213-2237.

[9] A. Bonito, J.-L. Guermond and F. Luddens, Regularity of the Maxwell equations in heterogeneous media and Lipschitz domains. J. Math. Anal. Appl. 408 (2013) 498-512.

[10] D. Braess and R. Verfuerth, A posteriori error estimators for the Raviart-Thomas element. SIAM J. Numer. Anal. 33 (1996) 2431-2444.

[11] S.C. Brenner, A multigrid algorithm for the lowest-order Raviart-Thomas mixed trangular finite element method. SIAM J. Numer. Anal. 29 (1992) 647-678.

[12] J. Bussac and P. Reuss, Traité de neutronique. Hermann (1985).

[13] P. Ciarlet Jr. E. Jamelot and F.D. Kpadonou, Domain decomposition methods for the diffusion equation with low-regularity solution. Comput. Math. Appl. 74 (2017) 2369-2384.

[14] M. Costabel, M. Dauge and S. Nicaise, Singularities of maxwell interface problems. ESAIM: M2AN 33 (1999) 627-649.

[15] M. Dauge, Benchmark Computations for Maxwell Equations. Available at: https://perso.univ-rennes1.fr/monique.dauge/core/ index.html (2018).

[16] J.J. Duderstadt and L.J. Hamilton, Nuclear Reactor Analysis. John Wiley \& Sons, Inc. (1976).

[17] A. Ern and J.-L. Guermond, Theory and Practice of Finite Elements. Springer-Verlag (2004).

[18] R.S. Falk and J.E. Osborn, Error estimates for mixed methods. RAIRO Anal. Numer. 14 (1980) 249-277.

[19] P. Fernandes and G. Gilardi, Magnetostatic and electrostatic problems in inhomogeneous anisotropic media with irregular boundary and mixed boundary conditions. Math. Models Methods Appl. Sci. 7 (1997) 957-991.

[20] T.-P. Fries and T. Belytschko, The extended/generalized finite element method: an overview of the method and its applications. Int. J. Numer. Methods Eng. 84 (2010) 253-304.

[21] L. Giret, Non-conforming Domain Decomposition for the Multigroup Neutron $S P_{N}$ Equations. Ph.D. thesis, EDMH, Université Paris-Saclay (2018).

[22] P. Grisvard, Elliptic Problems in Nonsmooth Domains. Pitman (1985).

[23] E. Jamelot and P. Ciarlet Jr. Fast non-overlapping Schwarz domain decomposition methods for solving the neutron diffusion equation. J. Comput. Phys. 241 (2013) 445-463.

[24] E. Jamelot, A.-M. Baudron and J.-J. Lautard, Domain decomposition for the $S P_{N}$ solver MINOS. Transp. Theory Stat. Phys. 41 (2012) 495-512.

[25] E. Jamelot Jr. P. Ciarlet, A.-M. Baudron and J.-J. Lautard, Domain decomposition for the neutron $S P_{N}$ equations, in 21 st International Domain Decomposition Conference. Vol. 98 of Lecture Notes in Computational Science and Engineering (2014) 677-685.

[26] J.-C. Nédélec, Mixed finite elements in $\mathbb{R}^{3}$. Numer. Math. 35 (1980) 315-341.

[27] J.E. Osborn, Spectral approximation for compact operators. Math. Comput. 29 (1975) 712-725.

[28] P.-A. Raviart and J.-M. Thomas, A mixed finite element method for second order elliptic problems, in Mathematical Aspects of Finite Element Methods. Vol. 606 of Lecture Notes in Mathematics. Springer (1977) 292-315.

[29] A. Sargeni, K.W. Burn and G.B. Bruna, Coupling effects in large reactor cores: the impact of heavy and conventional reflectors on power distribution perturbations, in PHYSOR 2014, Kyoto, Japan, Sept 28-Oct 3, 2014 (2014). 
[30] D. Schneider, F. Dolci, F. Gabriel et al., APOLLO3 ${ }^{\circledR}$ : CEA/DEN deterministic multi-purpose code for reactor physics analysis, in PHYSOR 2016, Sun Valley ID, USA, May 1-5, 2016 (2016).

[31] O. Steinbach, Numerical Approximation Methods for Elliptic Boundary Value Problems. Springer, New York (2008).

[32] M.F. Wheeler and I. Yotov, A posteriori error estimates for the mortar mixed finite element method. SIAM J. Numer. Anal. 43 (2005) 1021-1042. 